\title{
Multifunctional ZnO nanorod-reduced graphene oxide hybrids nanocomposites for effective water remediation: Effective sunlight driven degradation of organic dyes and rapid heavy metal adsorption
}

\author{
Kuglaur Shanmugam Ranjith ${ }^{\mathrm{a}, *}$, Palanisamy Manivel $^{\mathrm{b}}$, Ramasamy Thangavel Rajendrakumar ${ }^{\mathrm{c}}$, \\ Tamer Uyar ${ }^{\mathrm{a}, *}$ \\ ${ }^{a}$ Institute of Materials Science E Nanotechnology and UNAM-National Nanotechnology Research Center, Bilkent University, Ankara 06800, Turkey \\ ${ }^{\mathrm{b}}$ Centre for Nanoscience and Nanotechnology, School of Physics, Bharathidasan University, Tiruchirappalli 620 024, Tamil Nadu, India \\ ${ }^{\mathrm{c}}$ Department of Nanoscience and Technology, School of Physics, Bharathiar University, Coimbatore 641 046, Tamil Nadu, India
}

\section{H I G H L I G H T S}

- Feasible design and interface improvise the catalytic behavior of nanocomposites.

- Distinctive features enhances the structural functionality and carrier separation.

- Tunable electro negative functionality of rGO favors the absorption site over $\mathrm{ZnO}$.

- Vital role behind the light absorption were favored by rGO ratio with $\mathrm{ZnO}$.

\section{A R T I C L E I N F O}

\section{Article history:}

Received 20 March 2017

Received in revised form 15 May 2017

Accepted 16 May 2017

Available online 17 May 2017

\section{Keywords:}

Reduced graphene oxide (rGO)

$\mathrm{ZnO}$ nanorods

Nanocomposites

Visible photocatalysis

Heavy metal adsorption

\section{G R A P H I C A L A B S T R A C T}

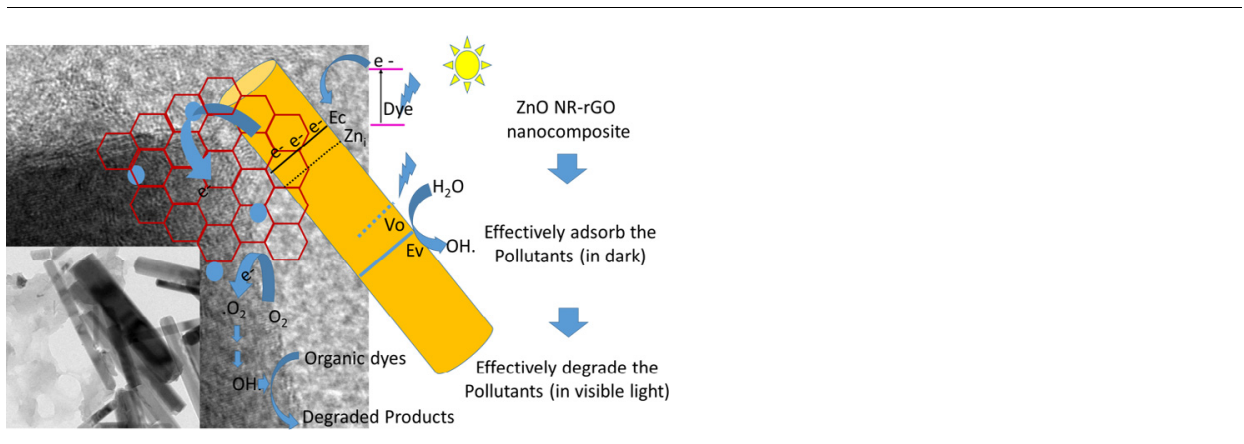

We demonstrate the multi-functionality engineering on nanocomposite by combining one dimensional (1D) ZnO nanorod (NR) and two dimensional (2D) reduced graphene oxide ( $\mathrm{rGO}$ ) for efficient water remediation. Nano-engineered $\mathrm{ZnO}$ NR-rGO nanocomposites show efficient water remediation in terms of degradation of organic dyes and removal of heavy metal ions. Herein, we report on the fabrication of ZnO NR-rGO nanocomposite via a facile template-free hydrothermal route with an aim to improve the visible photocatalytic efficiency of the ZnO NR based nanocomposites. The structural and morphological features reveal that the rGO sheets are attached on the $\mathrm{ZnO}$ NRs and form a hybrid composite assembly. The surface enabled ZnO NR-rGO nanocomposites were used to degrade organic dye molecules (methylene blue (MB), methyl orange (MO) and rhodamine $\mathrm{B}(\mathrm{RhB})$ ) under visible irradiation and adsorb $\mathrm{Cu}(\mathrm{II})$

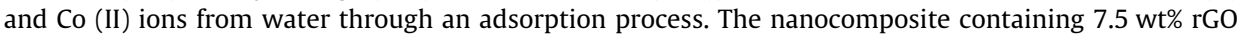
and $\mathrm{ZnO}$ NRs shows a 4-fold enhancement in the visible photocatalytic activity and effective removal of $\mathrm{Cu}$ (II) and $\mathrm{Co}$ (II) ions from aqueous solution respectively. The photocatalytic performance is discussed in detail with respect to interaction between $\mathrm{ZnO}$ NRs and rGO sheets, light-harvesting properties of the nanocomposites. The effective experimental adsorption data also fit very well with the pseudo-secondorder model which reveals the surface adsorption of metal ions. The results provide insight into a new method utilize for both visible photo degradation and adsorption for the removal of various wastewater pollutants. Construction of hybrid form of nanostructures delivers the effective catalytic properties with tunable functionalities for the water remediation.

(c) 2017 Elsevier B.V. All rights reserved.

\footnotetext{
* Corresponding authors.

E-mail address: uyar@unam.bilkent.edu.tr (T. Uyar).
} 


\section{Introduction}

Environmental water pollution caused by organic pollutants and heavy metals have become a serious problem worldwide that threatens the balance of nature and the sustainable endurance of human beings $[1,2]$. Most organic pollutants existing in trace or even ultra-trace quantities in the environment would be toxic and could accumulate along the food chain which may lead to serious ecological and health hazards. In order to eliminate the hazardous heavy metals and organic pollutants in wastewater, various photocatalytic semiconductor materials, such as $\mathrm{TiO}_{2}$, $\mathrm{ZnO}, \mathrm{CdS}, \mathrm{Fe}_{2} \mathrm{O}_{3}$ etc. were used in contiguous years [3,4]. Meanwhile, wideband gap semiconductors such as $\mathrm{TiO}_{2}$ and $\mathrm{ZnO}$ are promising photocatalysts but they hinder the effective absorption of visible light in photocatalytic process [5]. In order to bring the wide band gap semiconductor active in visible photo energy, various materials have been employed as effective change extractor through hybrid hetero structural interface [6]. One dimensional (1D) metal oxide nanostructured materials such as nanofibers, nanotubes, and nanorods are particularly interested as catalysts, owing to their large surface to volume ratio, efficient electron transport and ability to generate more reactive oxygen species on the surface [7]. Meanwhile, heavy metal removal on semiconducting nanostructures was investigated through colorimetric and fluorescence chemo sensing process [8,9]. Despite their independent advances, a system integrating both the rapid removal and effective degradation of organic pollutants remains largely unexplored. A rationally designed material with such feature could greatly enhance the versatility of the functional devices, improve the efficiency and reduce the water treatment cost. The fine grain size of the metal oxide nanostructures favor the aggregation by the Van der walls force or electrostatic interaction and it's minimize the adsorption ability, stability and selectivity [4,9]. To overcome this technical bottleneck, some researcher's immobilized nanometersized metal oxides on different carbon based materials such as activated carbon (AC), carbon nanotubes (CNTs) and graphene in order to improve its functional properties [10]. In the carbon family, graphene has an attractive two-dimensional single-layer sheet of $\mathrm{sp}^{2}$-hybridized carbon atoms, possesses excellent charge carrier and electron mobility, which leads to effective separation of photogenerated carriers with high surface area, excellent thermal conductivity, and high electronic conductivity, and optically transparency [11]. Recently, the combination of oxide semiconductor and graphene functionalities including $\mathrm{TiO}_{2}, \mathrm{ZnO}, \mathrm{CuO}, \mathrm{MnO}_{2}$,
$\mathrm{Fe}_{2} \mathrm{O}_{3}, \mathrm{CoFe}_{2} \mathrm{O}_{4}, \mathrm{MnFe}_{2} \mathrm{O}_{4}$ has been demonstrated to effectively improve the photocatalytic activities in these composite systems [12]. The primary role of graphene in these composite semiconductor photocatalyst systems is to delocalize the photogenerated electron through its $\pi$ network, which inhibits the recombination process and hence improves the photocatalytic performance. Chen et al. [13] indicated that carbon based iron oxide magnetic composites could be employed as an effective adsorbent for the adsorption of $\mathrm{Ni}(\mathrm{II})$ and $\mathrm{Sr}(\mathrm{II})$ from wastewater. The adsorption capacity of the composites was determined to be much higher than that of metal oxides. Graphene possesses similar physical properties to CNTs but has larger surface areas than the latter. Construction of photo active 1D nanostructures over the two dimensional (2D) carbon based structures offers the promising photoactivity and effective charge separation efficiency with improvised surface functionality. By grafting over the elongated 1D nanorods on 2D graphene nano sheet were furnish a new form of nanocomposite system and its offer a facile and continuous, directionated electron flow with numerous reaction sites, stability and highly efficient catalytic properties $[14,15]$.

In this current study, the $\mathrm{ZnO}$ nanorods (NRs) on reduced graphene oxide ( $\mathrm{rGO}$ ) nanocomposites ( $\mathrm{ZnO} \mathrm{NR}-\mathrm{rGO}$ ) were prepared by simple grafting of or growth of $\mathrm{ZnO}$ NRs in a homogeneously dispersed GO solution and subsequent drying (Scheme 1). ZnO NR-rGO samples were tested for degradation of the organic dye molecules such as methylene blue (MB), methyl orange (MO) and rhodamine $\mathrm{B}(\mathrm{RhB})$ and also for the adsorption of $\mathrm{Cu}$ (II), Co (II) ions from aqueous systems. This work not only demonstrated the possibility and validity of $\mathrm{ZnO}$ NR-rGO nanocomposite as promising adsorbents for pollutant removal and environmental remediation, but also gives insight into understanding the catalytic and adsorptive behavior of ZnO NR-rGO nanocomposite. We demonstrated that the grafted of $1 \mathrm{D}$ ZnO NR catalysts on both sides of a 2D rGO scaffold form a charge transportable network and result in enhanced catalytic activity and improved adsorptive nature. The kinetics of the process was also very well examined by fitting the experimental data with theoretical models.

\section{Experimental section}

\subsection{Materials}

Graphite flake, sodium nitrate $\left(\mathrm{NaNO}_{3}\right)$, potassium permanganate $\left(\mathrm{KMnO}_{4}\right)$, zinc nitrate hexahydrate $\left(\mathrm{Zn}\left(\mathrm{NO}_{3}\right)_{2} \cdot 6 \mathrm{H}_{2} \mathrm{O}\right)$,

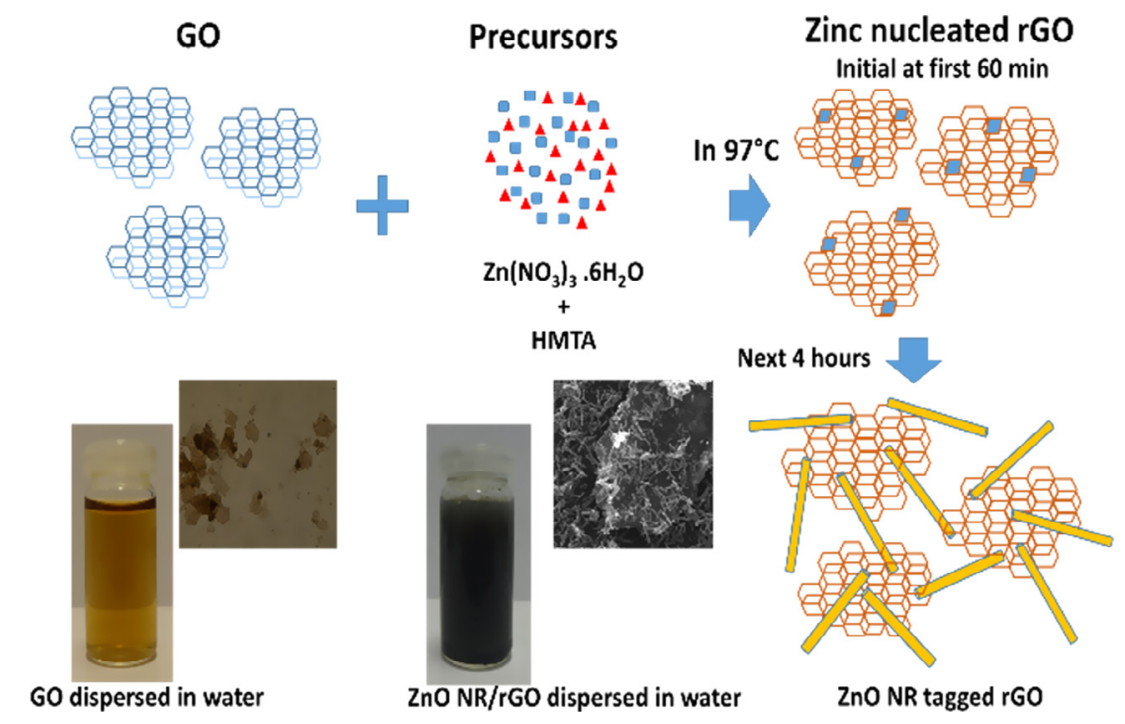

Scheme 1. Illustration of the synthesis processing of ZnO NR-rGO nanocomposite bridges. 
hexamethylenetetramine (HMTA), copper chloride $\left(\mathrm{CuCl}_{3}\right)$ and cobalt chloride $\left(\mathrm{CoCl}_{3}\right)$, rhodamine B (RhB), methyl orange (MO), methylene blue (MB), nitric acid $\left(\mathrm{HNO}_{3}\right)$, sulfuric acid $(95 \%$, $\left(\mathrm{H}_{2} \mathrm{SO}_{4}\right)$ ) and ethanol $\left(\mathrm{C}_{2} \mathrm{H}_{5} \mathrm{OH}\right)$ were purchased from Sigma Aldrich. Polyethyleneimine (PEI) were acquired from Scientific Chemical Reagent Co, Ltd. All chemicals were analytical grade. The solvent used in this study is deionized and doubly distilled water.

\subsection{Preparation of ZnO-rGO nanocomposites}

Graphene oxide (GO) was synthesized from natural graphite flakes by a modified Hummers method [16]. The 1D ZnO NRs were synthesised through the aqueous solution using equal mole of $(0.125 \mathrm{mM}) \mathrm{Zn}\left(\mathrm{NO}_{3}\right)_{2} \cdot 6 \mathrm{H}_{2} \mathrm{O}$ and HMTA [17]. The clear experimental procedures for synthesis of GO and ZnO NRs were given in supporting information part. In a typical synthesis, $20 \mathrm{~mL}$ of deionized water containing specific weight ratio of GO was added in ZnO NR growth precursor solution. After stirring for $30 \mathrm{~min}$, the mixed solution was transferred into a $100 \mathrm{~mL}$ autoclave with a Teflon liner, which was kept at $140^{\circ} \mathrm{C}$ for $4 \mathrm{~h}$ and then cooled to room temperature naturally. The products were rinsed thoroughly with distilled water and ethanol and annealed under nitrogen atmosphere at $150^{\circ} \mathrm{C}$ for $4 \mathrm{~h}$. Other samples were synthesized via the same procedure with different weight ratio of GO flakes to $\mathrm{ZnO}$ NRs. After the hydrothermal process, GO were thermally reduced and form the ZnO-rGO based nanocomposites. The as-obtained composites were labelled based on the weight ratio of $\mathrm{GO}$ to $\mathrm{ZnO}$ in precursor materials. For example, GZ2.5 means that the $2.5 \mathrm{wt}$ $\%$ of GO with $\mathrm{ZnO}(\sim 350 \mathrm{mg})$. As per that 2.5, 5.0, 7.5, 10.0, $12.5 \mathrm{wt} \%$ of GO added $\mathrm{ZnO}$ nanocomposites were named as GZ2.5, GZ5.0, GZ7.5, GZ10.0 and GZ12.5, respectively.

\subsection{Adsorption kinetics of dye molecules}

The batch mode adsorption studies for RhB were carried out by an agitating photocatalyst in dye solution at $25^{\circ} \mathrm{C}$. Typically, $50 \mathrm{~mL}$ of $\mathrm{RhB}(10 \mathrm{mg} / \mathrm{L})$ aqueous solution and $25 \mathrm{mg}$ of photocatalyst (ZnO, GZ7.5 and GO) were added into a beaker and agitated in the dark. Then, aliquots $(1 \mathrm{~mL})$ were withdrawn at specific time intervals to determine solution concentrations. The concentrations of various dyes were monitored using UV-vis absorption spectrophotometer at $525.5 \mathrm{~nm}$ for $\mathrm{RhB}$. The equilibrium adsorption capacities of dyes onto photocatalysts were determined according to the following formula:

$\mathrm{Qe}(\mathrm{mg} / \mathrm{g})=((\mathrm{Co}-\mathrm{Ce}) \mathrm{V}) / \mathrm{M}$

where Qe $(\mathrm{mg} / \mathrm{g})$ is the equilibrium adsorption capacity of the adsorbent, $C_{0}$ and $C_{e}(\mathrm{mg} / \mathrm{L})$ are the initial and final concentrations of dyes, respectively, $\mathrm{V}(\mathrm{L})$ is the volume of the original mixture, and $\mathrm{M}(\mathrm{g})$ is the weight of photocatalyst added.

\subsection{Photocatalytic degradation measurements}

The photocatalytic degradation experiments for $\mathrm{RhB}, \mathrm{MO}$, and MB were carried out in a self-prepared reactor. In the degradation procedure, $50 \mathrm{mg}$ catalysts were immersed in a $150 \mathrm{~mL}$ beaker containing $100 \mathrm{~mL}$ of $\mathrm{RhB}, \mathrm{MO}, \mathrm{MB}$ and aqueous solution $(10 \mathrm{mg} /$ $\mathrm{L}$ ). The solution was irradiated by a $75 \mathrm{~W}$ xenon lamp (visible light with UV cut off filters) and natural sunlight with UV cut off filter (Lee type 226 (USA)). For sunlight irradiation, samples were irradiated on a normal sunny day between 9.00 a.m. and 4.00 p.m under the shadows in order to avoid the evaporation of solution through heat effect. The light intensity was the constantly checked every 30 min using the lux meter. The adsorption-desorption equilibrium was attained by 50 min stirring in a dark room. The vertical distance between the solution level and the horizontal plane of the lamp was fixed at $10 \mathrm{~cm}$. At an interval of $30 \mathrm{~min}, 3 \mathrm{~mL}$ of solution was taken out from the reactor. The absorbance of the solution was determined using a UV-Vis absorption photometer at the characteristic absorption wavelength $(661 \mathrm{~nm}, 542.5 \mathrm{~nm}$ and $464.5 \mathrm{~nm}$ for MB, RhB and MO respectively). The degradation efficiency of dyes by the photocatalysts was determined according to the following formula:

$\eta \%=((\mathrm{Co}-\mathrm{Ce}) / \mathrm{Ce}) \times 100$

where $\eta(\%)$ is the degradation efficiency, $C_{0}$ and $C_{e}(m g / L)$ are the initial and final concentrations of dyes, respectively.

\subsection{Sorption studies}

The Cu (II) and Co (II) adsorption process was executed for pure $\mathrm{ZnO} \mathrm{NR}$ and $\mathrm{rGO}$ on $\mathrm{ZnO}$ adsorbents to determine the optimum adsorption conditions. A typical batch mode adsorption experiments were carried out with ZnO NR-rGO nanocomposite adsorbent at room temperature. The effect of $\mathrm{pH}$ on the adsorption capacity (Qo) was investigated in the range from 2 to 10 by using $\mathrm{Cu}$ (II) and Co (II) solution prepared from the standard $100 \mathrm{mg} / \mathrm{L}$ stock solution to the preferred concentration of $10 \mathrm{mg} / \mathrm{L}$ through sequential dilution. The $\mathrm{pH}$ was adjusted by using sodium hydroxide $(\mathrm{NaOH})$ or hydrochloric acid $(\mathrm{HCl})$ solutions. The resultant mixture solutions were transferred to $50 \mathrm{~mL}$ standard measuring flask and stirred on a thermostatic mechanical shaker operating at a constant speed of $300 \mathrm{rpm}$. Finally, the sample was withdrawn from the shaker at predetermined time intervals and the supernatant was collected by centrifuging at $5000 \mathrm{rpm}$ for $3 \mathrm{~min}$. The resultant clear solution was used to estimate the $\mathrm{Cu}$ (II) and $\mathrm{Co}$ (II) adsorption by using spectrophotometric analysis. The supernatant solution of $20 \mathrm{~mL}$ was taken in a test tube and $2 \mathrm{~mL}$ of polyethyleneimine solution $(0.94 \mathrm{mg} / \mathrm{mL})$ was added. This was further incubated for color development and finally the absorbance of the samples was measured in a UV-visible spectrophotometer in the wavelength of $275 \mathrm{~nm}$ and $315 \mathrm{~nm}$ for Cu (II) and Co (II) ions respectively [18].

\subsection{Materials characterizations}

The structure, microstructure and morphology of the as obtained samples were characterized by X-ray powder diffraction (PANalytical X'Pert multipurpose X-ray diffractometer) with $\mathrm{Cu}$ $\mathrm{K} \alpha$ radiation $(\lambda=1.54056 \AA)$, Raman spectroscopy (in Via-Reflex, Renishaw, U.K.), field-emission scanning electron microscopy (FESEM, Quanta, FEG 250), transmission electron microscopy (TEM, FEI-Tecnai G2 F30), Fourier transform infrared spectroscopy (VERTEX 801 HYPERION 2000, Bruker Optics, Germany). X-ray photoelectron spectroscopy (XPS, Thermo K-alpha-monochromated) was employed to analyze the surface chemical composition. The diffused reflectance and fluorescence spectra of the samples were taken at room temperature on a UV-visible spectrophotometer (UV3600, Shimadzu, Japan), and photoluminescence (PL) spectro fluorometer (time-resolved fluorescence spectrophotometer (FL1057 TCSPC) with an excitation at $325 \mathrm{~nm}$. The absorbance spectra of organic pollutants and heavy metal ions were obtained by using UV-vis Spectrophotometer (Varian Cary 100). The chromatic experiment with HPLC-UV/vis system were carried out on liquid chromatograph mass spectroscopy (Agilent Technologies 6224 TOF) using binary solvent gradient pump and automatic sample injector. The specific surface area of the samples were analysis using Brunauer-Emmet-Teller (BET) method carried out on Micromeritics Tristar analyser after degassing the samples for $3 \mathrm{~h}$ at $200{ }^{\circ} \mathrm{C}$. 


\section{Result and discussion}

\subsection{Structural and morphological studies}

The typical X-ray diffraction (XRD) patterns of the GO, ZnO NR and GZ composites (GO loading level: $2.5 \mathrm{wt} \%, 7.5 \mathrm{wt} \%$ and 12.5 wt\%, respectively) were shown in Fig. 1. It can be observed from the XRD pattern that the as prepared GO shows a sharp peak at $2 \theta=10.6^{\circ}$, corresponding to the $(001)$ reflection of GO. From Fig. 1, all the diffraction peaks of the pure $\mathrm{ZnO}$ and $\mathrm{ZnO}$ NR-rGO composites (GO loading level: $2.5 \mathrm{wt} \%, 7.5 \mathrm{wt} \%$ and $12.5 \mathrm{wt} \%$ ) synthesized can be matched with hexagonal ZnO (JCPDS 36-1451). In the case of ZnO NR-rGO, the major diffraction peaks are consistent with pure $\mathrm{ZnO}$ phase and the broad diffraction peak assigned at $24.5^{\circ}$ is attributed to the (002) plane of typical rGO. The d (002) peak near $24.5^{\circ}$ is related to the stacking layers along the c-axis of reduced graphene oxide [19]. And it indicates that GO is simultaneously reduced to reduced graphene oxide at the temperature influence during the formation of $\mathrm{ZnO}$ NRs

Fig. 2 shows the FESEM and TEM images of as prepared GO, pure ZnO NRs and sample GZ7.5. Inset of the Fig. 2a shows the optical image of the GO reveal that the flakes with average size distribution of 5-10 $\mu \mathrm{m}$ and were observed to be composed of a few layers (Supportive information, Fig. S1). FESEM image of graphene oxide (Fig. 2a) shows the folded nature of the sheets. Fig. 2b shows the TEM image of few layered, wrinkled and folded GO. Insert shows the SAED of GO with diffused diffraction ring pattern which represents the disordered structure of GO due to the chemical oxidation on the conjugated graphite structure. In Fig. 2c, the hydrothermally

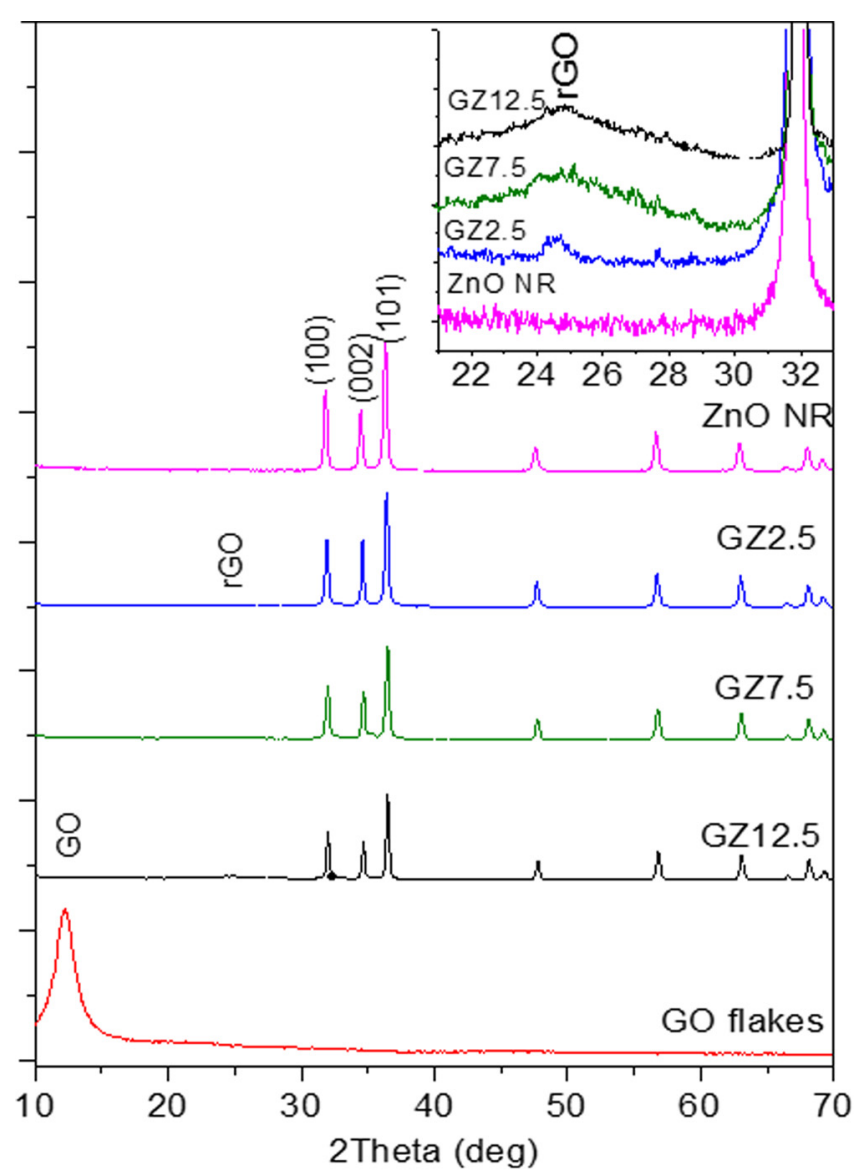

Fig. 1. The XRD patterns of ZnO NR, GO, GZ2.5, GZ7.5 and GZ12.5 nanocomposite samples. grown $\mathrm{ZnO}$ NRs are in the prismatic form, with length of $2-3 \mu \mathrm{m}$ and the diameter of $100 \mathrm{~nm}$. High-resolution transmission electron microscopy (HRTEM) lattice fringes of a single NR shows the oriented growth along the $c$ axis [0001] direction with lattice spacing of $0.26 \mathrm{~nm}$ representing $(002)$ axis [20]. FESEM and TEM images of GZ7.5 reveal a well-defined attachment of ZnO NRs on rGO flakes which reveal the formation of $\mathrm{ZnO}$ NR-rGO nanocomposite from submicrometer to several micrometers (Fig. 2e). In the meantime, the ZnO NRs in the GZ7.5 are viewed to be wrapped in the interior of the pore walls which consists of thin layers of stacked rGO sheets (Fig. 2f). TEM observation further confirms the even distribution of $\mathrm{ZnO}$ with the wrapped interface with rGO sheets (Fig. 3).

Fig. 3 shows the TEM images of GZ7.5 which reveals that the ZnO NRs are intimately attached on the rGO sheet surface and distributed, with no morphological changes after the combination with rGO sheets via hydrothermal treatment. From the TEM images, it shows the presence of wrinkles and ripples and scrolls on the $\mathrm{ZnO} \mathrm{NR}$ and rGO suggests the occurrence of few layered rGO sheets. Inset of the Fig. $3 \mathrm{~b}$ and $\mathrm{c}$, spotted pattern of the selected area electron diffraction (SAED) pattern of rGO reveal that rGO, employed as a reference, was observed to be composed of a few layers [21] (Fig. S2). As a function of initial hydrothermal process, $\mathrm{ZnO}$ nuclei were attached over the rGO and exhibit the presence of $\mathrm{ZnO}$ NRs progressively grown on the rGO flakes (Fig. S3 and Fig. 2f) which reveals the nucleation sites anchoring the ZnO NR on the rGO flakes during the growth process. However, SAED image of rGO showed set of six-fold symmetric diffraction points of a typical hexagonal configuration represents the thermal reduction of GO [19]. The specific BET surface area of the ZnO NR and ZnO NRrGO (GZ7.5) nanocomposites were determined to be $2.336 \mathrm{~m}^{2} / \mathrm{g}$ and $19.434 \mathrm{~m}^{2} / \mathrm{g}$, respectively. From the comparative isotherm results (Fig. S4), the decoration of rGO on the ZnO NRs apparently leads to an increase in the surface area as compared to the pristine ZnO NRs.

Raman spectroscopy was utilized to assess the existence and crystalline quality of rGO sheet in the ZnO NR-rGO nanocomposite (Fig. 4). For all composite specimens, two prominent characteristic peaks were observed at 334 and $435 \mathrm{~cm}^{-1}$, correspondingly assigned as $E_{2}^{\text {high }}-E_{2}^{\text {low }}$ and $E_{2}^{\text {high }}$ modes of the crystalline $\mathrm{ZnO}$, respectively [22]. The peak at $582 \mathrm{~cm}^{-1}$ is assigned to (LO) mode, attributed to oxygen deficiency defects in $\mathrm{ZnO}$ [23]. The peak at $1130 \mathrm{~cm}^{-1}$ is due to the multiple-phonon scattering processes [24]. The intensity of these peaks is reduced in composites as compared to that in $\mathrm{ZnO}$ due to the interaction between $\mathrm{ZnO}$ and rGO. In addition, at higher wavelength the peak should observed at $\sim 1140 \mathrm{~cm}^{-1}$ assigned to the 2LO vibrations which is due to the second order raman process [25]. Fig. 4(b-d) shows the Raman spectrum of GO and GZ composites. The $\mathrm{G}$ band at $1577 \mathrm{~cm}^{-1}$ is assigned to the E2g phonon mode of $C$ sp2 atoms and the D band at $1336 \mathrm{~cm}^{-1}$ is a breathing mode of k-point phonons of A1g symmetry, originating from the defects. The intensity ratio $\left(\mathrm{I}_{\mathrm{D}} / \mathrm{I}_{\mathrm{G}}\right)$ of the $\mathrm{D}$ band to the $\mathrm{G}$ band of GO is 0.801, which is the measure of Raman vibrations, expressed by the sp3/sp2 carbon ratio. For the ZnO NR-rGO hybrid composite samples, two broad peaks corresponding to the $D$ and $G$ band and the band intensity ratio was estimated as $\sim 1.06$. The peak intensity ratio were consistent with typical rGO, indicating that rGOs coexisted with ZnO NRs. Compared to GO, the hybrids exhibit a slight increase in the $\mathrm{I}_{\mathrm{D}} / \mathrm{I}_{\mathrm{G}}$ ratio, which is due to the presence of more defects and the interaction between rGO and ZnO NRs [26]. The results indicate that $\mathrm{ZnO} \mathrm{NR}-\mathrm{rGO}$ nanocomposite were successfully prepared which was correlated with XRD results.

\subsection{Optical studies}

The UV-vis DRS absorption spectra of the ZnO NR-rGO nanocomposites are shown in Fig. S5a. The pure ZnO shows a sharp 

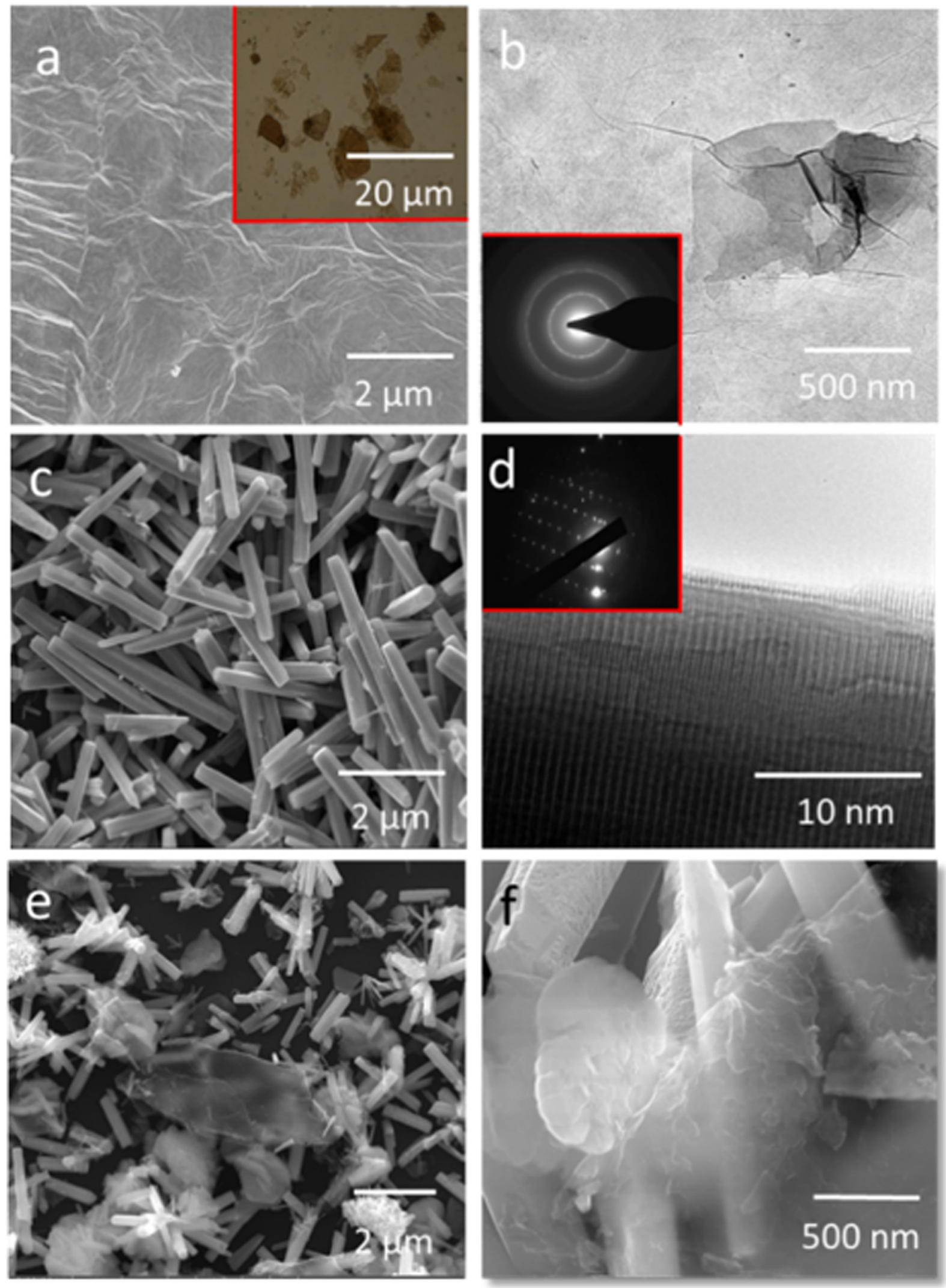

Fig. 2. FESEM (a) and TEM (b) images of GO synthesized by modified Hummers method. The insert of (a) and (b) corresponds to the optical images and SAED pattern of GO, respectively. SEM (c) and TEM (d) images of the ZnO NR formed by the hydrothermal process. The insert in (d) is the corresponding SAED pattern of ZnO NR. FESEM (e) image of GZ7.5 and high magnification FESEM (f) image of GZ7.5 nanocomposites. 

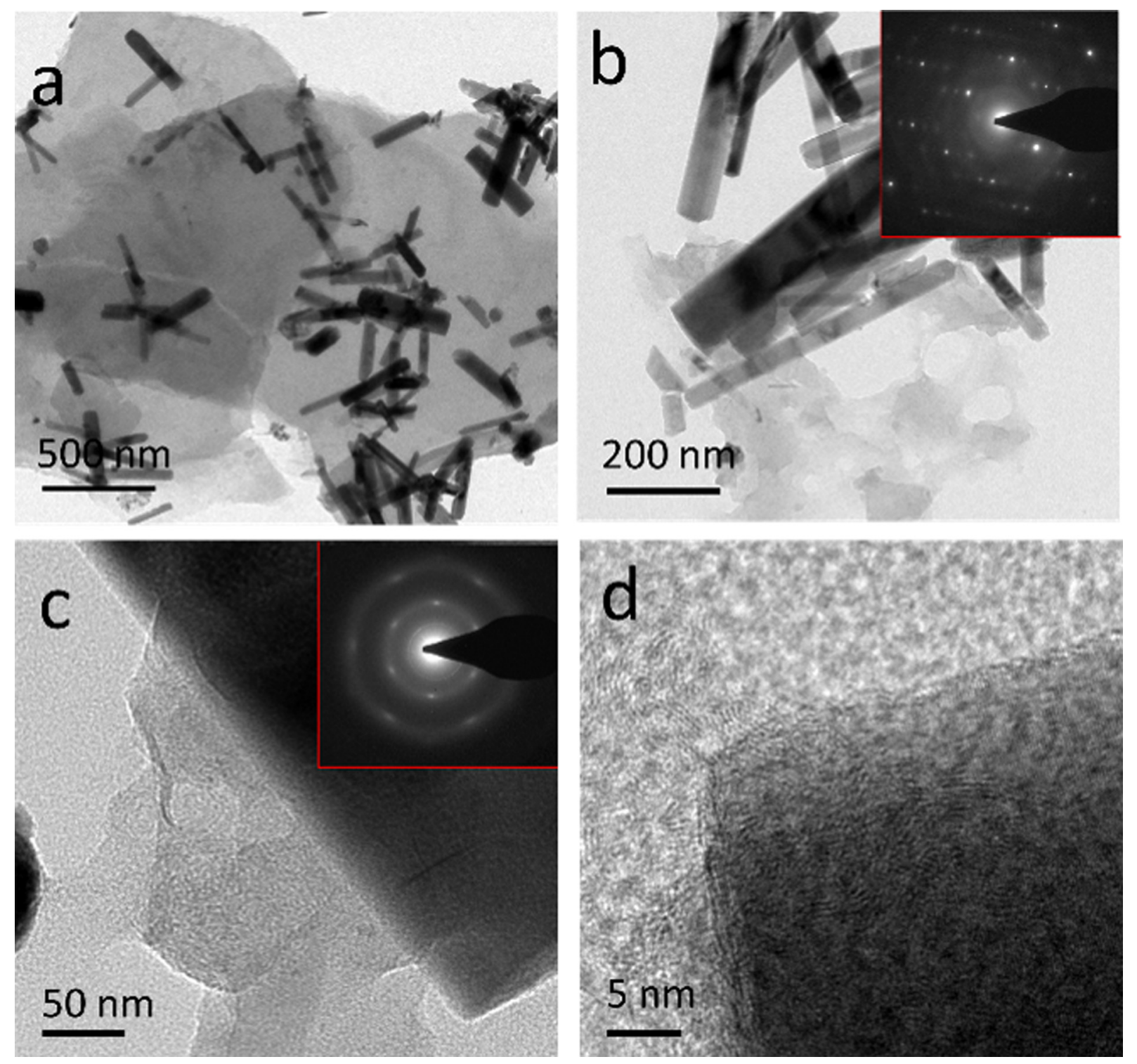

Fig. 3. (a, b) TEM and (c, d) HRTEM micrographs of the GZ7.5 nanocomposites. Inset show the SAED of the respective images.

absorption at $384 \mathrm{~nm}$ and the ZnO NR-rGO nanocomposite show the enhanced absorption in the visible region $(400-800 \mathrm{~nm})$ in addition to the absorption edge at $380 \mathrm{~nm}$. The $\mathrm{ZnO}$ NR-rGO hybrids possess an intense and broad range of absorption in the visible region due to the presence of rGO. Compared to pure $\mathrm{ZnO}$, the absorption intensity of GZ samples are reduced. With the increased mass ratios of rGO sheets, the absorption background of $\mathrm{ZnO}$ NR-rGO nanocomposite increase in the visible light range, which is ascribed to the black body effects of rGO sheets [26]. PL spectra provide useful information for the investigation of the interface charge carrier transfer and photogenerated electron-hole recombination process in $\mathrm{ZnO}$ NR-rGO nanocomposite system. Fig. 5 shows the room temperature photoluminescence spectra of pure and hybrid composites. All the samples exhibits the prominent sharp emission at $369 \mathrm{~nm}$ and $387 \mathrm{~nm}$ which attributes to the band edge emission and the $\mathrm{Zn}$ interstitial trap states related band below the conduction band and a broad emission at $550 \mathrm{~nm}$ which related to the oxygen related defect states [27]. The raised broad violet emission around $419 \mathrm{~nm}$ is probably due to the radiational defect related to the interfacial traps existing at the interface and emitted the radiation transition. The broad emission at $550 \mathrm{~nm}$ assigned to the trap-state emission result from the intrinsic defects and vacancies of $\mathrm{ZnO}$, such as interstitial oxygen and oxygen vacancies [28]. Hydrothermally grown ZnO NRs have the broad and promising defect related emission which was found to be composed of two major Gaussian components (Fig. S6) centered at 540 and $580 \mathrm{~nm}$. The small 540 and $580 \mathrm{~nm}$ component can be related to the singly and doubly charged oxygen vacancy states $\left(\mathrm{Vo}^{+}\right.$and $\left.\mathrm{Vo}^{++}\right)$, respectively [29]. Compared with pure ZnO NRs, a significant depression in the PL emission was observed on introducing the rGO with the $\mathrm{ZnO}$ as the form of ZnO NR-rGO nanocomposite. This depression became more noticeable as the rGO mass ratio increased, suggesting the successful electron transfer from $\mathrm{ZnO}$ to $\mathrm{rGO}$ with the suppressed recombination process. This phenomenon supports the argument that the rGO acted as an effective electron scavenger for $\mathrm{ZnO}$, leading to the pronounced charge separation observed for the ZnO NR-rGO nanocomposite. Decrease in the fluorescence yield of composite; suggest an additional pathway for the disappearance of charge carriers because of the interaction between excited $\mathrm{ZnO}$ NRs and rGO sheets. For the higher wt\% of ZnO NR-rGO nanocomposite, emission peaks in the violet region are observed. It may due to trap state emission over the interface which promotes the $\mathrm{Zn}$ interstitial based defect emission or the peaks related due to the decay of exci- 


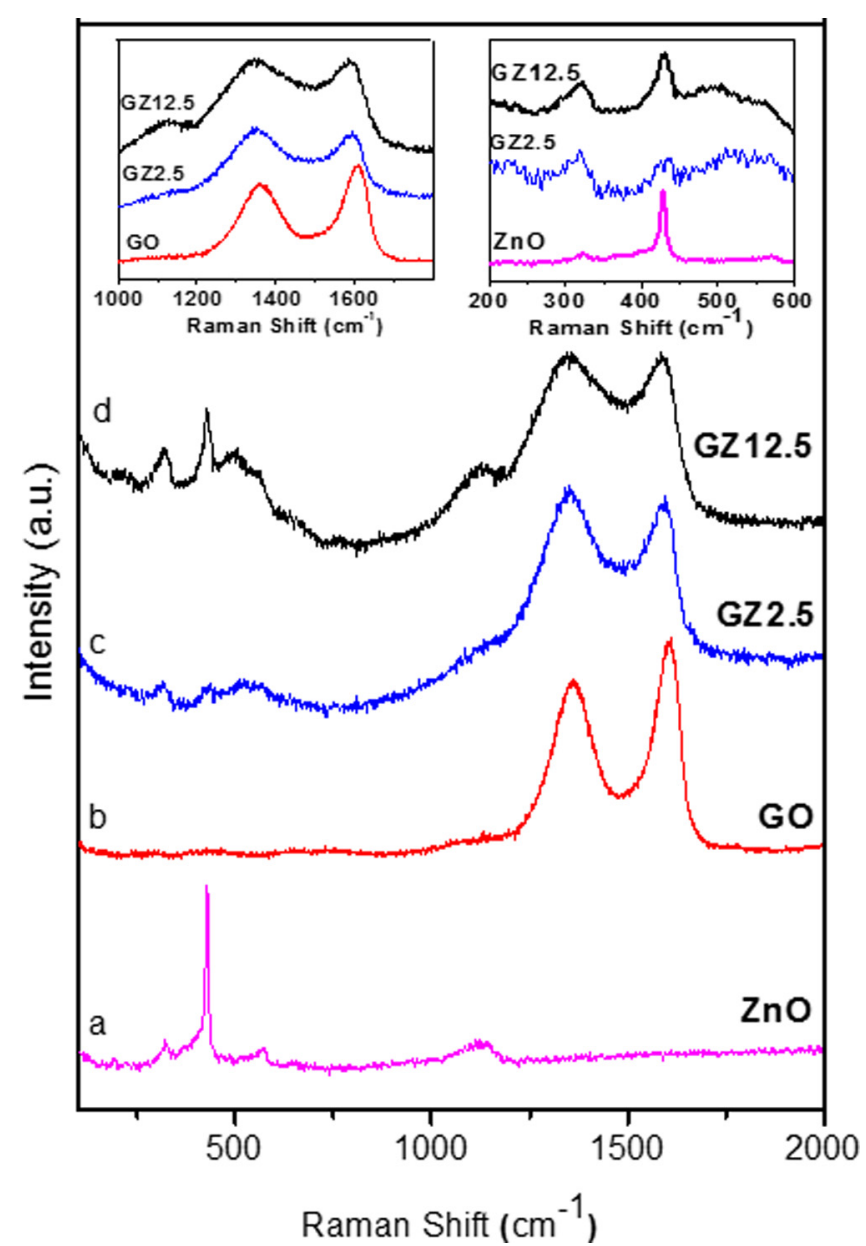

Fig. 4. Raman spectra of ZnO NR, GO, GZ2.5 and GZ12.5 nano composite samples.

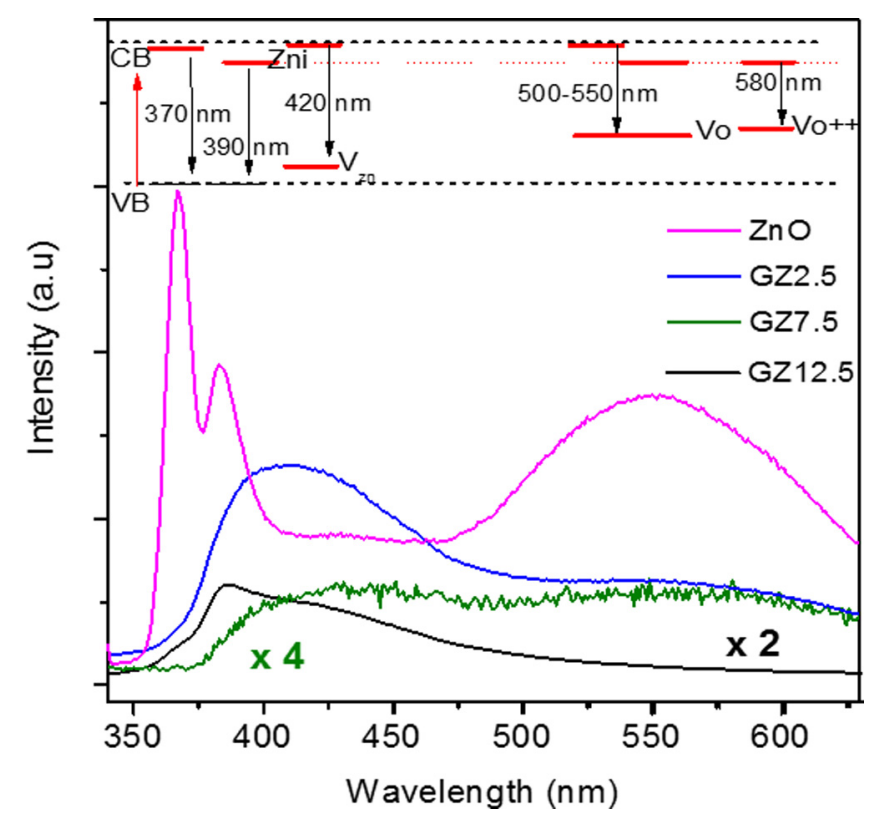

Fig. 5. Photoluminescence spectra of $\mathrm{ZnO}$ NR-rGO hybrid nanocomposites compared to pure ZnO NRs synthesized by hydrothermal method.

tons [30] from the LUMO of the graphene layer with an epoxy bond to the $\mathrm{ZnO}$ valence band.

The XPS full-scan survey spectrum shows the principal C1s, O1s, and $\mathrm{Zn} 2 \mathrm{p}$ core levels in Fig. 6a. Inset shows the high resolution $\mathrm{Zn}$
$2 \mathrm{p} 3 / 2$ peak position of $\mathrm{ZnO} \mathrm{NR}$ and GZ7.5 nanocomposites and the $\mathrm{Zn} 2 \mathrm{p}^{3 / 2}$ and $\mathrm{Zn} 2 \mathrm{p}^{1 / 2}$ main peaks with a difference of $23.1 \mathrm{eV}$ shown in the Fig. S7a imply the characteristic peaks of $\mathrm{Zn}^{2+}$ [28]. However the shift to a higher binding energy by the $0.8 \mathrm{eV}$ signifies the increase in the oxidation state of $\mathrm{Zn}$, resulting from the contact with the electronegative reduced graphene [31]. This indicated the strong dipole interaction between the electro negative rGO and ZnO NRs. By comparing the peak intensity of 01 s between $\mathrm{ZnO}$ and GZ samples, which reveal that the defect levels were turned on after tagging the $\mathrm{ZnO}$ and $\mathrm{GO}$ (Fig. 6b). By fitting the oxygen related band in the $\mathrm{ZnO} \mathrm{NR}-\mathrm{rGO}$ nanocomposite system by Gaussian function, it has been split into 4 set of peaks such as $\mathrm{O}$ ion in $\mathrm{ZnO}$ lattice $\left(\mathrm{O}_{\mathrm{L}}\right)(530.1 \mathrm{eV}), \mathrm{O}$ ions in oxygen-deficient lattice in $\mathrm{ZnO}\left(\mathrm{O}_{\mathrm{V}}\right)(531.1 \mathrm{eV})$, chemisorbed oxygen species $\left(\mathrm{O}_{\mathrm{C}}\right)$ $(532.4 \mathrm{eV})$ and band interaction of $\mathrm{C}-\mathrm{O}$ in $\mathrm{rGO}$ at $533.4 \mathrm{eV}$. Hydrothermally synthesized $\mathrm{ZnO}$ NRs have prominent $\mathrm{O}_{\mathrm{V}}$ ratio which is equivalent to the $\mathrm{O}_{\mathrm{L}}$. While decorating the rGO with $\mathrm{ZnO}, \mathrm{O}_{\mathrm{V}}$ ratio reduced due to the oxidization of $\mathrm{ZnO}$ by the electronegative rGO with the increased functionality of chemisorbed oxygen species. A peak at $533.42 \mathrm{eV}$ is observed for $\mathrm{ZnO}$ NR-rGO nanocomposite, which is attributed to the $\mathrm{C}-\mathrm{O}$ band in $\mathrm{rGO}$.

The reduction of GO after the hydrothermal process was further confirmed by the XPS C1 s spectra (Fig. 6c). As shown in Fig. 6c, the C $1 \mathrm{~s}$ XPS spectra of GO and GZ7.5 contained four deconvolution peaks at 284.5, 286.7, 287.5 and $289.1 \mathrm{eV}$, which represented $\mathrm{C}-\mathrm{C}, \mathrm{C}-\mathrm{O}, \mathrm{C}=\mathrm{O}$ and $\mathrm{O}-\mathrm{C}=\mathrm{O}$ bonding, respectively. In brief, from the C1s XPS spectra of GO (Fig. 6c), a considerable degree of oxidation with four components that corresponds to carbon atoms in different functional groups can be seen: the non-oxygenated ring $\mathrm{C}$, the $\mathrm{C}$ in $\mathrm{C}-\mathrm{O}$ bonds, the $\mathrm{C}$ in $\mathrm{C}=\mathrm{O}$ bonds and the carboxylate carbon $(\mathrm{O}-\mathrm{C}=\mathrm{O})[32]$. After hydrothermal process with the ZnO NRs, a notable decrease in oxygen content is clearly visible; in addition, the peak corresponding to the $\mathrm{C}=\mathrm{O}$ bond has disappeared. The oxygen loss mainly results from the suppression of $\mathrm{C}-\mathrm{O}$ peak, and the relative ratio of $\mathrm{C}(\mathrm{O}) \mathrm{OH}$ decreases remarkably as well. For the GZ7.5, an essentially low O-bound C content was obtained (23.91\% for GZ7.5), as compared with the as prepared GO flakes (approximately 49.12\%) indicating that the oxygen containing groups were significantly removed in the hydrothermal process [33]. More importantly, the low O-bound C content of the present ZnO NR-rGO nanocomposite ensures the occurrence of pronounced charge separation, which is crucial to its resultant photocatalytic performance. Fig. 6d shows the FTIR spectra of GO, ZnO NR, and GZ7.5. The broad absorption band at $3425 \mathrm{~cm}^{-1}$ is assigned to the hydroxyl groups of absorbed $\mathrm{H}_{2} \mathrm{O}$ molecules. The absorption band at $450 \mathrm{~cm}^{-1}$ of GZ7.5 is similar to that of pure $\mathrm{ZnO}$, which is attributed to stretching models of $\mathrm{Zn}-\mathrm{O}$. There is an obvious decrease in the intensities of oxygen functional group peaks in $\mathrm{GO}$ centered at $1071 \mathrm{~cm}^{-1}$ (C-O stretching), $1182 \mathrm{~cm}^{-1}(\mathrm{C}-\mathrm{O}-\mathrm{H}$ deformation vibrations), and $1730 \mathrm{~cm}^{-1}$ ( $\mathrm{C}=\mathrm{O}$ stretching of $\mathrm{COOH}$ groups) vibration peaks in GZ7.5 compared to those in GO, indicating reduction of oxygen-containing groups of $\mathrm{GO}$ in the $\mathrm{ZnO}$ NRrGO nanocomposite system [34]. The peak at $1615 \mathrm{~cm}^{-1}$ can be assigned to the skeletal vibrations of unoxidized graphitic domains. However, all these bands related with the oxygen functional groups almost disappear or appear with significantly lower intensities in the FTIR spectra of the GZ nanocomposites. These results confirm that these oxygen functional groups are almost removed in the process of thermal functionalization, and the GO is reduced to a great extent.

\subsection{Photocatalytic degradation}

Pure ZnO NRs exhibit a nearly negligible adsorption (less than $5 \%$ in $3 \mathrm{~h}$ ) for organic pollutants (Fig. 7a). For GO the adsorption efficiency for MB, RhB and MO are 27.8, 23.3, and 17.3\% after $3 \mathrm{~h}$ 

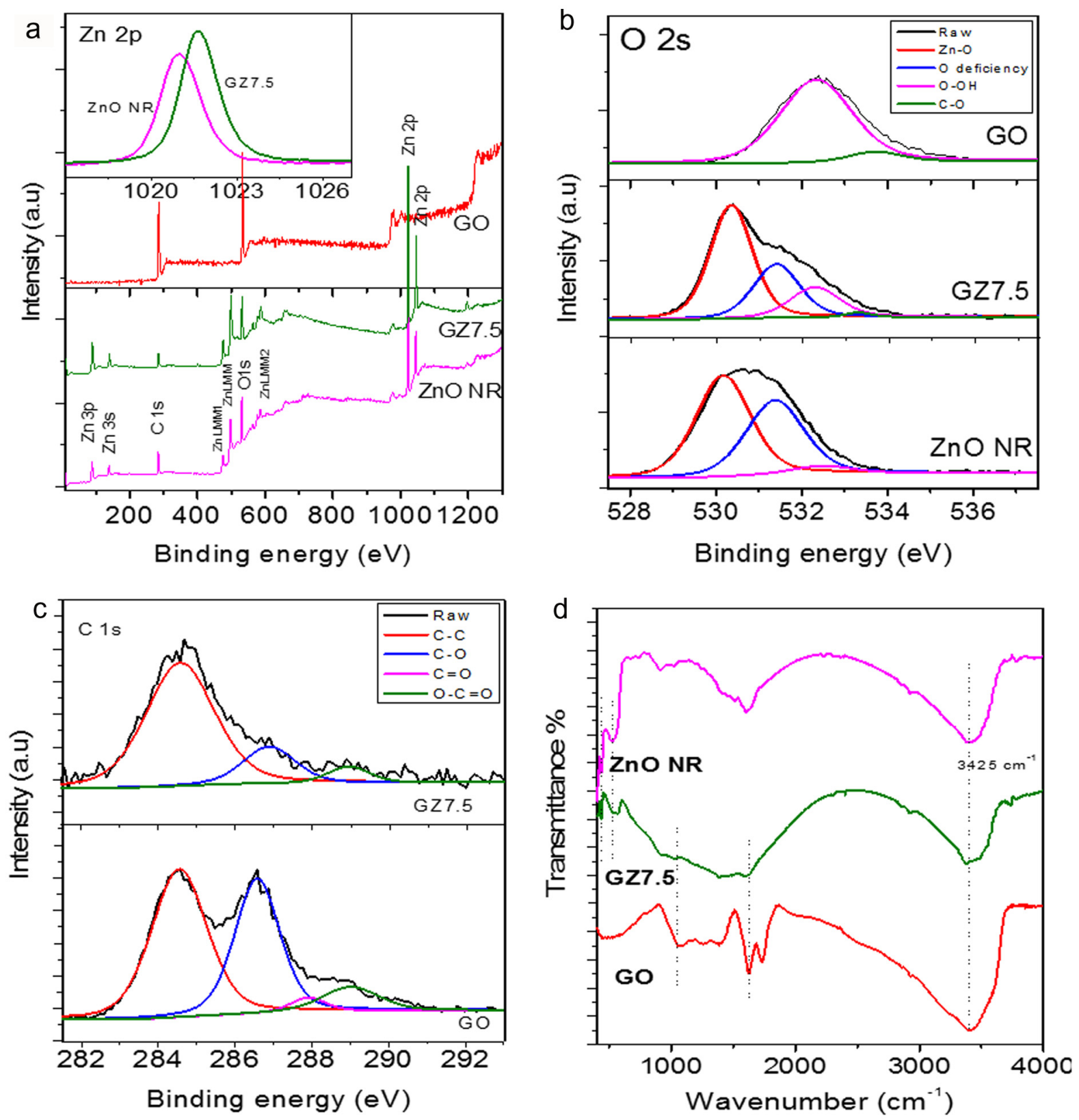

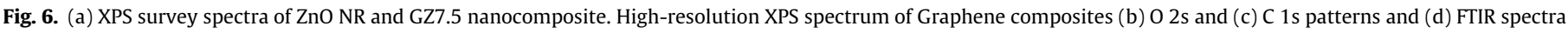
of $\mathrm{ZnO} \mathrm{NR}, \mathrm{GZ7.5}$ and GO respectively.

and the GZ12.5 samples propose the adsorption rates for MB, RhB and $\mathrm{MO}$ are $15.8,13.3$, and $10.2 \%$ respectively, (Fig. $7 \mathrm{a}$ ) all of which are much higher than those of pure ZnO NRs. After the introduction of rGO, the adsorption rates are greatly enhanced due to its higher specific surface area. Prior to irradiation, the photocatalytic reaction system was magnetically stirred in the dark for 50 min to reach the adsorption/desorption equilibrium of dye molecules on the surface of the photocatalysts. Fig. 7b and c shows the degradation rate of $\mathrm{RhB}$ with $\mathrm{ZnO}$ and $\mathrm{GZ}$ composites under visible and natural sunlight irradiation, respectively. After $100 \mathrm{~min}$ of visible irradiation, the photocatalytic degradation efficiency of RhB for ZnO NR, GZ2.5, GZ5.0 GZ7.5 GZ10.0 and GZ12.5 are 25.9, 49.6, 59.8, 80.4, 43.5 and 37.2\%, respectively, and except ZnO NR, all other GZ composites were promisingly active under visible irradiation. Clearly, GZ7.5 shows superior photo degradation rate (Fig. S8) of $0.0227 \mathrm{~min}^{-1}$ and $0.0258 \mathrm{~min}^{-1}$ under visible and natural sunlight irradiation which is 7 times higher than the pure
ZnO NRs. Fig. 7d shows that the photo degradation efficiency of $\mathrm{MB}, \mathrm{RhB}$ and MO for sample GZ7.5 is $93.9,88.08$ and $75.3 \%$ after 180 min of irradiation, much higher than those of pure ZnO NR. This indicates that the introduction of rGO is greatly beneficial to the improvement of catalytic activity by work as a sensitizer in the photoreaction. For further study on photocatalytic mechanism (Fig. S9 $(\mathrm{a}-\mathrm{c})$ ) shows the batch experiment of photodegradation of $\mathrm{MB}, \mathrm{RhB}$ and $\mathrm{MO}$ (initial concentration, $10 \mathrm{mg} / \mathrm{L}, 100 \mathrm{~mL}$ ) with $50 \mathrm{mg}$ of $\mathrm{ZnO}$ and GZ7.5 for various durations. About $80 \%$ of pollutants were removed in 90 min after visible illumination in presence of GZ7.5.

In contrast, only $20 \%$ was removed in presence of $\mathrm{ZnO}$ NRs. The degradation profile of different pollutants reveals that the cationic dyes such as MB and RhB interacted more with the hybrid system than $\mathrm{MO}$ and results in faster efficient degradation. This was due to the higher negative potential value in hybrid composites which favors the more absorbance rate for the cationic dyes than the 

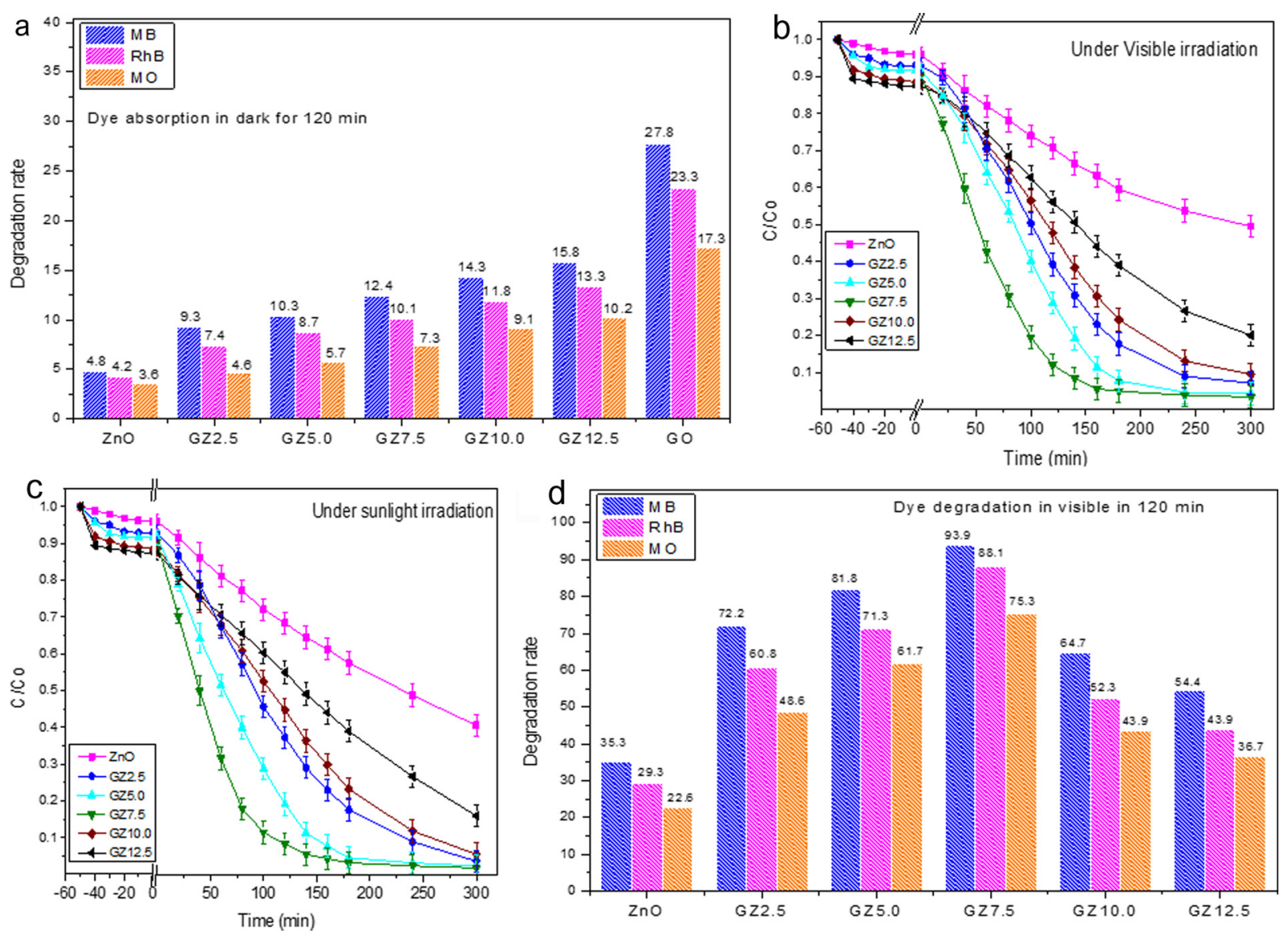

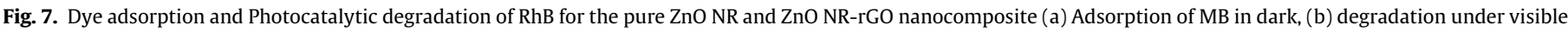
irradiation, (c) degradation under sunlight irradiation and (d) comparative degradation efficiency over different dye pollutants.

anionic dye, MO. In the cycling experiments for the photodegradation of RhB using GZ7.5, the degradation rate is as high as $92.4 \%$ after 10 cycles (Fig. S10), suggesting great stability of the photocatalyst. Additionally, as a control experiment catalytic performance of GZ7.5 were compared with the $7.5 \mathrm{wt} \%$ of GO-ZnO mixed composites (Fig. S11). The mixed form of catalyst exhibits the slower catalytic efficiency as compared with GZ7.5 which clearly reveals the contract interface and carrier transport between ZnO NRs and rGO are crucial factors to achieve higher photocatalytic activities. The dominant factor in order to improve the catalytic activity was that while grafting the ZnO NRs on the rGO surface, continuous conductive paths are built through the rGO, thereby effectively reducing the transfer resistance of photogenerated electrons and inhibiting the recombination of photogenerated electrons and holes. Further, on inducing the ZnO NR-rGO nanocomposite system, created interfacial electric field between the $\mathrm{ZnO}$ and rGO, thus improving the separation efficiency of photo induced electron-hole pairs. Due to the natural functionality of higher electron transfer ability in graphene based system, the lifetime of photo induced electrons and holes in the hybrid system improves. This indicates that it has great application potential nature.

While increasing the rGO dosage over GZ7.5, the catalytic efficiency starts to depress in the GZ10.0 and GZ12.5. This decrease in the catalytic behavior is believed to be due to overloading of rGO that act as a block that avoid the light absorption of $\mathrm{ZnO}$ and encourages the electron hole recombination [33]. Even though the effective absorption rate was high over the rGO dosage, decrease in the carrier separation efficiency results in the suppression of resultant catalyst and it may called as shield effect. Similar behavior on the effect of rGO dosage has been reported previously, where excess of rGO caused a negative influence over the catalytic performance [35]. Additionally the dye molecules which we used has come sensitization effect which will give some charge carrier to the catalytic surface which can promotes the radical production to degrade the dye molecules under visible illumination [36]. But the possibility over the subsequent electron transfer from the excited dyes to the $\mathrm{ZnO}$ would be determined by the adsorption of dyes through the electrostatic interaction with the catalytic surface. At the neutral $\mathrm{pH}, \mathrm{ZnO}$ has positively charged (point of zero charge of $\mathrm{ZnO}$ is at around $\mathrm{pH}_{\mathrm{zpc}} \sim 9$ ) and electrostatically it repels the cationic dyes. But the presence of rGO with the $\mathrm{ZnO}$ will favor the dye interaction and induces the possibility of sensitization effect. And according to the dosage of rGO with $\mathrm{ZnO}$ the dye absorption rate were increased in contrast to degradation rate (Fig. 7a). It reveals that, there is a possibility of dye sensitization effect but the effective degradation probabilities were determined by the carrier separation efficiency at the $\mathrm{ZnO}$ and rGO interface under visible irradiation.

On the basis of the above analyses, we propose that the improved photocatalytic activity of composites mainly results from the interaction of $\mathrm{ZnO}-\mathrm{rGO}$ owing to the more efficient separation of electron-holes, and the improved optical absorption in visible light range. A possible reaction mechanism and the photo degradation process are illustrated in Fig. 8. Irradiated by the simulated visible photon energy, excited electrons in the $\mathrm{ZnO}$ NRs were extracted by the rGO bridges and high conductivity of rGO sheets enable quick charge transfer, thereby prolonging the lifetime of charge carriers and suppressing the recombination. The photoinduced electrons on $\mathrm{ZnO}$ surface and the trapped electrons on rGO sheets induce redox reaction and degrade the organic pol- 


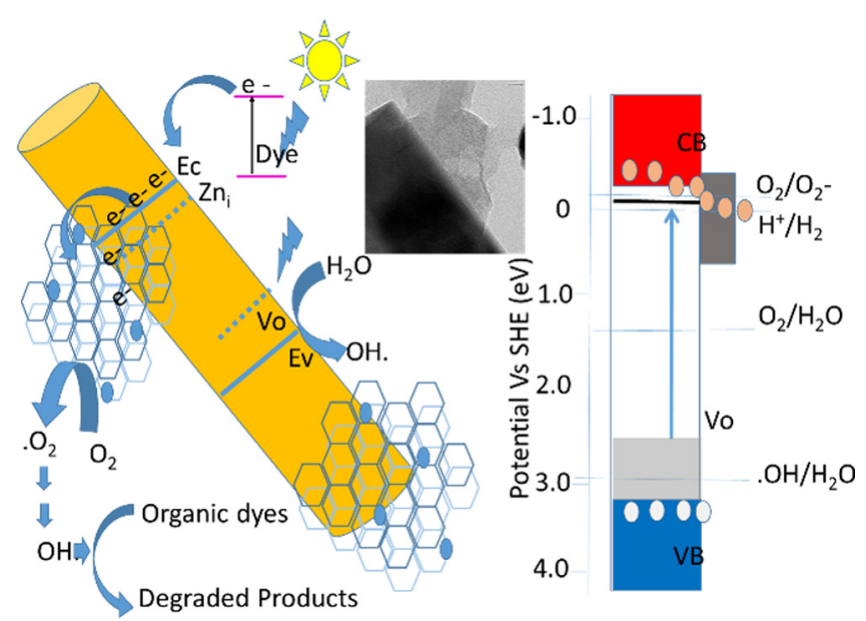

$\mathrm{pH} 6.0$

Fig. 8. Schematic and mechanism of photocatalytic degradation using ZnO NR-rGO nanocomposite hybrids.

lutants. Several mechanisms have been proposed to account for the photocatalytic behavior on the inorganic-organic based photocatalytic system $[8,9,13,14,37]$. We believe that excellent performance of GZ7.5 can be attributed by the aspects of both the surface interaction over the surface adsorbed molecules and the effective transfer of charge carriers. The visible catalytic ability and favorable higher interaction of rGO on the ZnO NR surface were investigated by analysing the degradation performance of the colorless 4cholorophenol which provides evidences for the excluded dye sensitization effect and denotes the visible catalytic behavior through the rGO-ZnO nanocomposite formation (Fig. S12).

In general, dye based pollutant degradation mechanism could be explained by the electron-hole $\left(\mathrm{e}-\mathrm{h}^{+}\right)$separation between conduction and the valance band and the slow recombination rate of charge carriers in the $\mathrm{ZnO}$ system. During photo irradiation, separated carriers promoted the production of reactive oxygen species such as active hydroxyl $\left(\mathrm{OH}\right.$.) and superoxide $\left(\mathrm{O}_{2}\right)$ radicals. These $\mathrm{OH}$ and $\mathrm{O}_{2}$ have been considered as oxidative species and usually determine the degradation of various organic dyes under photo irradiation. Based on the proposed degradation mechanism, higher effective charge separation of ZnO NR-rGO nanocomposite sufficiently produces active oxygen radicals which are basically responsible for the degradation of organic dye molecules. Meanwhile, the rGO supported ZnO NR composite catalysts exhibits higher visible photocatalytic degradation efficiency than the ZnO NR. This may be due to separation of efficient carriers on the surface by rGO in the composite, thus delaying the recombination rate and effectively producing sufficient amount of $\mathrm{O}_{2}$ species under photo irradiation. It is already reported that the oxygen radicals are produced as a result of rGO being grafted on the $\mathrm{ZnO}$ under photo irradiation and availability of sufficient amount of $\mathrm{O}_{2}$ species followed by $\mathrm{OH}$. production favors effective degradation of organic pollutants. Therefore, the heterogeneous catalyst ZnO NR-rGO nanocomposite network exhibits high degradation over organic dyes under visible light irradiation due to the generation of oxy-radicals on the surface. Further surface interaction of organic pollutants with $\pi-\pi$ conjunction over the aromatic regions of rGO increases the adsorption rate of dye molecules than the pure $\mathrm{ZnO}$ NR surface. And these adsorbed pollutant molecules were more favorable in terms of interaction with reactive oxygen species than those in the solution form. The promoted interfacial electric field between the $\mathrm{ZnO} \mathrm{NR}$ and rGO facilitates the separation of electro hole pairs and induced the faster carrier migration. And to further investigate the degradation sequence and the by-product formation during the catalytic process, the degradation of MB dye molecules respective to time under visible irradiation with $\mathrm{ZnO}$ and GZ7.5 were analysed through the LC-MS measurements. LC-MS spectra (Fig. S13) of the degraded dye solution with respective to time clearly reveals the degradation of $\mathrm{MB}$ dye molecules without forming any by products. After the irradiation of $180 \mathrm{~min}$, the toxic dye molecules in the aqueous solution were completely degraded on using GZ7.5 as the catalyst.

\subsection{Cu (II) and Co (II) adsorption}

As the ZnO NR-rGO nanocomposite have the ability to adsorb organic pollutants, their efficiency in effective adsorption to remove heavy metals and hazardous materials from wastewater are investigated. ZnO NR and ZnO NR-rGO (GZ7.5) nanocomposites were employed for the $\mathrm{Cu}$ (II) and $\mathrm{Co}$ (II) adsorption ability in aqueous solution medium. The removal efficiency of the $\mathrm{ZnO}$ NR and ZnO NR-rGO nanocomposite were studied by varying the $\mathrm{pH}$, initial concentration of the metal ions and contact time. Fig. 9a shows the effect of pH on the adsorption of $\mathrm{Cu}$ (II) and $\mathrm{Co}$ (II) ions from aqueous solution. The $\mathrm{Cu}$ (II) and $\mathrm{Co}$ (II) adsorption behavior of ZnO NR and $\mathrm{ZnO}$ NR-rGO nanocomposite was investigated by varying the $\mathrm{pH}$ from 2 to 10 in the adsorbent concentration of $100 \mathrm{mg} / \mathrm{L}$ with initial concentration of adsorbate as $20 \mathrm{mg} / \mathrm{L}$ and contact time of $180 \mathrm{~min}$. The results show the adsorption capacity of $\mathrm{Cu}$ (II) is $7.1,21.3,63.3,77.1$ and $68.5 \%$ for $\mathrm{ZnO} \mathrm{NR}$ in the $\mathrm{pH}$ of $2,4,6,8$, 10 respectively. For the $\mathrm{ZnO}$ NR-rGO (GZ7.5) nanocomposite bridges the adsorption efficiency was 9.7, 49.7, 96.4, 98.6 and $96.9 \%$ for the $\mathrm{pH}$ of $2,4,6,8,10$ respectively.

Further investigations were carried out using Co (II) as representative. Results revealed that the adsorption percentage of Co (II) increased from $11.7 \%$ to $90.1 \%$ on varying the $\mathrm{pH}$ from 2 to 10 over the ZnO NR-rGO nanocomposite. The effect of contact time on the adsorption of metal ions was studied under neutral $\mathrm{pH}$ by adding $10 \mathrm{mg} / \mathrm{L}$ of adsorbent and $20.0 \mathrm{~mL}$ of sample solution into special glass stoppered tubes and the experiments were performed when the contact time varied from 10 to $120 \mathrm{~min}$. Results were shown in Fig. 9b. It is evident that the adsorption of metal ions on ZnO NR-rGO nanocomposite is initially very fast (Fig. S14). In the present work, $120 \mathrm{~min}$ was selected as the contact time to ensure equilibrium. Sorption kinetics is an important aspect for defining the adsorption efficiency and is expressed in terms of the rate of solute removal that controls the residence time of the adsorbate in the solid-solution interface [38]. The kinetics of the removal of $\mathrm{Cu}$ (II) and Co (II) ions was determined in order to understand the adsorption behavior of the GZ composites. Fig. S15 shows the adsorption data of $\mathrm{Cu}$ and $\mathrm{Co}$ ions by GZ composites at different time intervals. And the adsorption rate over the heavy metal ions was high as compared to the dye molecules over the ZnO NR and GZ7.5 nanocomposites (Fig. S16).

The kinetic data for the sorbent were fitted to a pseudo-first and second-order kinetic model and the calculated parameters from two kinetic models were listed in Table S1. The pseudo-secondorder equation provides better correlation coefficient and agreement between calculated qe values. The results indicate that $\mathrm{Cu}$ (II) and Co (II) uptake onto GZ composites are favorable by the pseudo-second-order kinetic model and it reveals that the chemical adsorption can be well described with the pseudo-second-order kinetic model (Fig. S15b) [39].

\subsection{Adsorption isotherms}

The equilibrium adsorption isotherm was investigated in order to describe the interactive behavior between adsorbate and adsorbent, and also for designing adsorption systems [40]. Adsorption data were studied by Langmuir and Freundlich isotherm models 

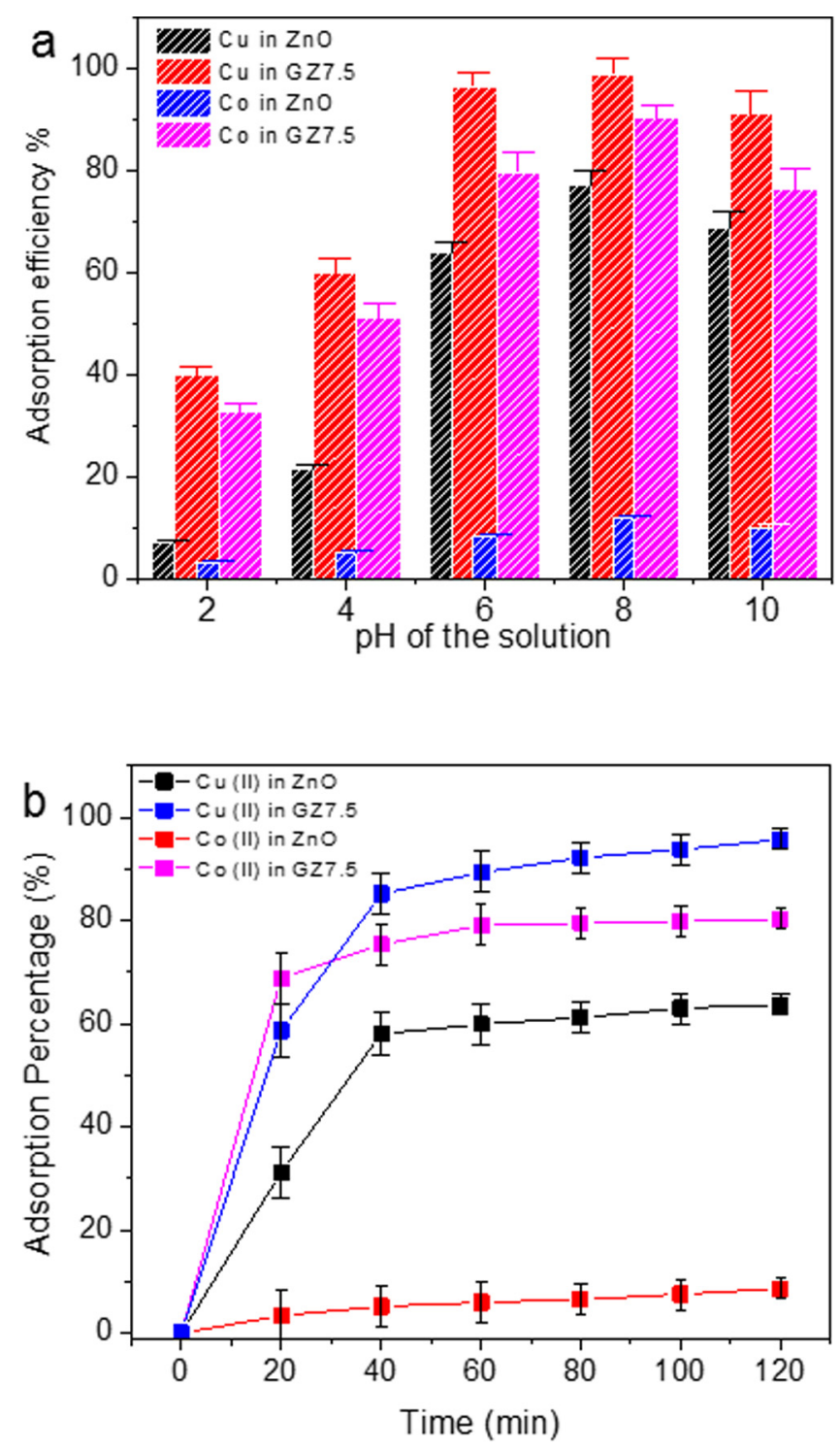

Fig. 9. (a) Effect of solution $\mathrm{pH}$ on the adsorption (Dosage of adsorbent $=10 \mathrm{mg}$, concentration of metal ions $=10 \mathrm{mg} / \mathrm{L}$, contact time $=120 \mathrm{~min}$.) (b) Effect of contact time on the adsorption of $\mathrm{Cu}$ (II), and Co (III) onto ZnO NR and GZ7.5 composites. (Dosage of adsorbent $=10 \mathrm{mg}$, concentration of metal ions $=10 \mathrm{mg} / \mathrm{L}, \mathrm{pH}=6.0$ ).

when the initial metal ion concentrations ranged from 10 to $100 \mathrm{mg} / \mathrm{L}$. The results obtained from the two adsorption isotherms are shown in Table S2. By comparing the $\mathrm{R}^{2}$ values of both models, the Langmuir model was seen to fit the experimental data better, suggesting monolayer adsorption of $\mathrm{Cu}$ (II), and Co (II) onto the surface of the ZnO NR-rGO nanocomposite. Furthermore, the values of the dimensionless parameter, $\mathrm{R}_{\mathrm{L}}$, are between 0.0812 and $0.792(0<\mathrm{RL}<1)$, which is consistent with the requirement for a favorable adsorption process [41]. The mapping and EDAX results reveal that the presence of $\mathrm{Cu}$ and $\mathrm{Co}$ ions over the GZ composite after adsorption concluded the adsorption of metal ions over the composite surface (Fig. S18). Mapping results from the Fig. S18 reveals the presence of $\mathrm{Cu}$ over the $\mathrm{ZnO}$, indicating that the $\mathrm{ZnO}$ can absorb the $\mathrm{Cu}$ ions and while grafting the rGO with $\mathrm{ZnO}$ enhances the $\mathrm{Cu}$ metal ion absorption. This may be due to the strong electronegativity of $\mathrm{Cu}^{2+}$ ion that can bond with $\mathrm{O}$ atom over the $\mathrm{ZnO}$ nanostructures [42]. But this trend was not observed with the Co metal ions, and in this case, rGO plays the sole role for the adsorption process. We could see the extent of metal adsorption from the elemental distribution/mapping of $\mathrm{Co}$ and $\mathrm{Cu}$ over
Table 1

Comparison of maximum adsorption capacities $(\mathrm{mg} / \mathrm{g})$ with various adsorbents for $\mathrm{Cu}$ (II) and Co (II).

\begin{tabular}{|c|c|c|c|}
\hline Absorbents & $\begin{array}{l}\mathrm{Cu}(\mathrm{II}) \\
\mathrm{mg} / \mathrm{g}^{-1}\end{array}$ & $\begin{array}{l}\text { Co (II) } \\
\mathrm{mg} / \mathrm{g}^{-1}\end{array}$ & Ref \\
\hline Nanometer TiO2 & 26.50 & & {$[44]$} \\
\hline Kaolin & 0.76 & 0.919 & {$[45,46]$} \\
\hline Functionalized MWCNT & 6.64 & & [47] \\
\hline Amine functionalize magnetic nano absorbent & 12.43 & & {$[48]$} \\
\hline GO-CS aerogel & 25.4 & & [49] \\
\hline Amination graphene oxide & & 116.35 & {$[50]$} \\
\hline Magnetite-reduced graphene oxide & & 0.71 & [51] \\
\hline $\mathrm{G}-\mathrm{ZnO}$ & 37.45 & & [10] \\
\hline ZnO NR-rGO nanocomposites & 67.39 & 36.35 & This work \\
\hline
\end{tabular}

the ZnO NR-rGO nanocomposite, which proves the metal removal capability. The comparison of the maximum adsorption capacity of $\mathrm{Cu}$ (II), and Co (II) with various adsorbents is listed in Table S2. It can be seen that ZnO NR-rGO nanocomposite have a relatively large adsorption capacity of 67.399 and $36.354 \mathrm{mg} / \mathrm{g}$ for $\mathrm{Cu}$ (II) and Co (II) respectively, indicating that ZnO NR-rGO nanocomposite is a potential material for removal of $\mathrm{Cu}$ (II) and Co (II) from aqueous solutions. The Freundlich constant $\mathrm{n}$ is found to be greater than 1 which is a favorable condition for adsorption [43]. The maximum adsorption efficiency (Qo) observed based on Langmuir model was $67.399 \mathrm{mg} / \mathrm{g}$ for the ZnO NR-rGO nanocomposite with the correlation coefficient $\left(R^{2}\right)$ of 0.9872 as shown in Fig. S17. Data fitted with Freundlich isotherm and the values of adsorption isotherm constants $1 / \mathrm{n}$ and $\mathrm{R}^{2}$ are 0.1413 and 0.9083 respectively and the empirical parameter $1 / \mathrm{n}$ value is in the range $0.1<1 / \mathrm{n}<1$ which confirms that the adsorption process is favourable [43].

The Freundlich isotherm is favourable for adsorption of $\mathrm{Cu}(\mathrm{II})$ and $\mathrm{Co}(\mathrm{II})$ ions from aqueous solution by ZnO NR-rGO nanocomposite and confirms their good heterogeneous adsorption capacity. The $\mathrm{Cu}$ (II) and Co (II) removal capacity of the ZnO NR-rGO nanocomposite was higher than that of the reported values as presented in Table 1. Interestingly, on coupling the heavy metal adsorption and degradation of dye molecules under visible irradiation, the heavy metal adsorbed catalysts exhibited pronounced degradation performance which is 1.7 times faster than the catalytic activity without the heavy metal ions (Fig. S19). The adsorbed heavy metal ions on the catalyst surface favour the effective photo generated carrier separation rate which enhanced the production of reactive oxygen species and fastened the degradation rate. The coupling effect of heavy metal adsorption and photodegradation properties and their advantage over the effective carrier separation will be further investigated in our future works. The ZnO NR-rGO nanocomposite provides large adsorption sites due to their high specific surface area and the rGO exhibits the high adsorption capacity due to the $\pi-\pi$ interaction with the metal ions. Therefore, the adsorption capacities were relatively more and $\mathrm{Cu}$ (II) and Co (II) ions were easily adsorbed on the surface of the nanocomposites.

\section{Conclusion}

In summary, we successfully synthesized 1D ZnO NRs immobilized on rGO via hydrothermal process via anchoring ZnO NRs on rGO as an efficient catalyst for degradation and heavy metal adsorption. The reduction of GO during the hydrothermal process provided excellent dispersion in solution, thereby enabling uniform attachment of $\mathrm{ZnO}$ NRs along with their 1D axis without restacking of rGO. The unique attachment of the composite formation exhibited high catalytic degradation efficiency under visible light irradiation and stable dye and heavy metal adsorptive behav- 
ior compared to ZnO NRs. This was mainly attributed to (i) improved bifunctional catalytic activity; (ii) facile electron transport via rGO connected parallel with elongated 1D ZnO NRs; (iii) the high surface area of both rGO and ZnO NRs. Our synthesis strategy can be applied to diverse combinations using various types of catalytic metal oxides with reduced form of 2D GO. The unique morphological nature and complementary properties of the $1 \mathrm{D}$ metal oxide/2D carbon can open up new nanocomposite building blocks to develop highly efficient catalysts.

\section{Acknowledgements}

K.S. Ranjith acknowledge The Scientific \& Technological Research Council of Turkey (TUBITAK), BIDEB 2216-Fellowships for Research Fellowship Programme for Foreign Citizens) for postdoctoral fellowship. T. Uyar acknowledges The Turkish Academy of Sciences - Outstanding Young Scientists Award Program (TUBAGEBIP)-Turkey for partial funding.

\section{Appendix A. Supplementary data}

Supplementary data associated with this article can be found, in the online version, at http://dx.doi.org/10.1016/j.cej.2017.05.105.

\section{References}

[1] J.T. Kim, M.H. Son, D.H. Lee, W.J. Seong, S. Han, Y.S. Chang, Partitioning behavior of heavy metals and persistent organic pollutants among fetomaternal bloods and tissues, Environ. Sci. Technol. 49 (2015) 7411-7422.

[2] H. Kuang, C. Xing, C. Hao, L. Liu, L. Wang, C. Xu, Rapid and highly sensitive detection of lead ions in drinking water based on a strip immunosensor Sensors (Basel) 13 (2013) 4214-4224.

[3] P.Z. Raya, H.J. Shipley, Inorganic nano-adsorbents for the removal of heavy metals and arsenic: a review, RSC Adv. 5 (2015) 29885-29907.

[4] M. Hua, S. Zhang, B. Pan, W. Zhang, L. Lv, Q. Zhang, Heavy metal removal from water/wastewater by nanosized metal oxides: a review, J. Hazard. Mater. 211112 (2012) 317-331.

[5] J. Li, J. Zhang, L. Fang, J. Wang, M. Shen, X. Su, Enhanced visible light photocatalytic properties of $\mathrm{TiO}_{2}$ thin films on the textured multicrystalline silicon wafers, J. Mater. Chem. A 3 (2015) 4903-4908.

[6] Y. Wang, Q. Wang, X. Zhan, F. Wang, M. Safdar, J. He, Visible light driven type II heterostructures and their enhanced photocatalysis properties: a review, Nanoscale 5 (2013) 8326-8339.

[7] M. Ge, C. Cao, J. Huang, S. Li, Z. Chen, K. O. Zhang, S.S. Al-Dey, Y. Lai, A review of one-dimensional $\mathrm{TiO}_{2}$ nanostructured materials for environmental and energy applications, J. Mater. Chem. A 4 (2016) 6772-6801.

[8] A. Senthamizhan, A. Celebioglu, S. Bayir, M. Gorur, E. Doganci, F. Yilmaz, T. Uyar, Highly fluorescent pyrene-functional polystyrene copolymer nanofibers for enhanced sensing performance of TNT, ACS Appl. Mater. Interfaces 7 (2015) 21038-21046.

[9] Y. Liu, Y. Ma, C. Liu, Z. Zhang, W. Yang, S. Nie, X. Zhou, The effective removal of $\mathrm{Cr}(\mathrm{VI})$ ions by carbon dot-silica hybrids driven by visible light, RSC Adv. 6 (2016) 68530-68537.

[10] A. Cazetta, O. Pezoti, K.C. Bedin, T.L. Silva, A.P. Junior, T. Asefa, V.C. Almeida, Magnetic activated carbon derived from biomass waste by concurrent synthesis: efficient adsorbent for toxic dyes, ACS Sustainable Chem. Eng. 4 (2016) 1058-1068.

[11] Y. Zhu, S. Murali, W. Cai, X. Li, J.W. Suk, J.R. Potts, R.S. Ruoff, Graphene and graphene oxide: synthesis and properties and application, Adv. Mater. 22 (2010) 3906-3924.

[12] X. Xie, K. Kretschmera, G. Wang, Advances in graphene-based semiconductor photocatalysts for solar energy conversion: fundamentals and materials engineering, Nanoscale 7 (2015) 13278-13292.

[13] C.L. Chen, J. Hu, D.D. Shao, J.X. Li, X.K. Wang, Adsorption behavior of multiwall carbon nanotube/iron oxide magnetic composites for $\mathrm{Ni}(\mathrm{II})$ and $\mathrm{Sr}(\mathrm{II})$, J. Hazard. Mater. 164 (2009) 923-928.

[14] W.H. Ryu, T.H. Yoon, S.H. Song, S. Jeon, Y.J. Park, I.D. Kim, Bifunctional composite catalysts using $\mathrm{Co}_{3} \mathrm{O}_{4}$ nanofibers immobilized on nonoxidized graphene nanoflakes for high-capacity and long-cycle $\mathrm{Li}-\mathrm{O}_{2}$ batteries, Nano Lett. 13 (2013) 4190-4197.

[15] H.D. Lim, K.Y. Park, H. Song, E.Y. Jang, H. Gwon, J. Kim, Y.H. Kim, M.D. Lima, R. Ovalle Robles, X. Lepro, R.H. Baughman, K. Kang, Enhanced power and rechargeability of a $\mathrm{Li}_{-} \mathrm{O}_{2}$ battery based on a hierarchical-fibril CNT electrode, Adv. Mater. 25 (2013) 1348-1352.

[16] P. Manivel, M. Dhakshnamoorthy, A. Balamurugan, N. Ponpandian, D. Mangalaraj, C. Viswanathan, Conducting polyaniline-graphene oxide fibrous nanocomposites: preparation, characterization and simultaneous electrochemical detection of ascorbic acid, dopamine and uric acid, RSC Adv. 3 (2013) 14428-14437.

[17] K.S. Ranjith, R. Pandian, E. McGlynn, R.T. Rajendra Kumar, Alignment, morphology and defect control of vertically aligned $\mathrm{ZnO}$ nanorod array: competition between "surfactant" and "stabilizer" roles of the amine species and its photocatalytic properties, Cryst. Growth Des. 14 (2014) 2873-2879.

[18] J. Jiaa, A. Wua, S. Luan, Spectrometry recognition of polyethyleneimine towards heavy metal ions, Colloids Surf., A 449 (2014) 1-7.

[19] A. Ghosh, S. Shukla, G.S. Khosla, B. Lochab, S. Mitra, Sustainable sulfur-rich copolymer/graphene composite as lithium-sulfur battery cathode with excellent electrochemical performance, Sci Rep. 6 (2016) 25207.

[20] R. Das, A. Kumar, Y. Kumar, S. Sen, P.M. Shirage, Effect of growth temperature on the optical properties of $\mathrm{ZnO}$ nanostructures grown by simple hydrothermal method, RSC Adv. 5 (2015) 60365-60372.

[21] N.R. Wilson, P.A. Pandey, R. Beanland, R.J. Young, I.A. Kinloch, L. Gong, Z. Liu, K. Suenaga, J.P. Rourke, S.J. York, J. Sloan, Graphene oxide: structural analysis and application as a highly transparent support for electron microscopy, ACS Nano 3 (2009) 2547-2556.

[22] A. Calzolari, M.B. Nardelli, Dielectric properties and Raman spectra of $\mathrm{ZnO}$ from a first principles finite-differences/finite-fields approach, Sci Rep. 3 (2013) 2999.

[23] J.Y. Li, H. Li, Physical and electrical performance of vapor-solid grown $\mathrm{ZnO}$ straight nanowires, Nanoscale Res. Lett. 4 (2009) 165-168.

[24] X.Y. Ye, Y.M. Zhou, Y.Q. Sun, J. Chen, Z.Q. Wang, Preparation and characterization of $\mathrm{Ag} / \mathrm{ZnO}$ composites via a simple hydrothermal route, J. Nanopart. Res. 11 (2009) 1159-1166.

[25] T. Pandiyarajan, R.V. Mangalaraja, B. Karthikeyan, S.S. Guzman, H.D. Mansilla, D. Contreras, N. Escalona, M.A.G. Pinilla, Microstructure, vibrational and visible emission properties of low frequency ultrasound $(42 \mathrm{kHz})$ assisted $\mathrm{ZnO}$ nanostructures, RSC Adv. 6 (2016) 20437-20446.

[26] M.K. Kavitha, H. John, P. Gopinath, R. Philip, Synthesis of reduced graphene oxide-ZnO hybrid with enhanced optical limiting properties, J. Mater. Chem. C 1 (2013) 3669-3676.

[27] F. Kayaci, S. Vempati, I. Donmez, N. Biyikli, T. Uyar, Role of zinc interstitials and oxygen vacancies of $\mathrm{ZnO}$ in photocatalysis: a bottom-up approach to control defect density, Nanoscale 6 (2014) 10224-10234.

[28] A. Senthamizhan, B. Balusamy, Z. Aytac, T. Uyar, Grain boundary engineering in electrospun $\mathrm{ZnO}$ nanostructures as promising photocatalysts, Cryst. Eng. Commun. 18 (2016) 6341-6351.

[29] A. Janotti, C.G. Van de Walle, Native point defects in ZnO, Phys. Rev. B. 76 (2007) 165202

[30] D.I. Son, B.W. Kwon, D.H. Park, W.S. Seo, Y. Yi, B. Angadi, C.L. Lee, W.K. Choi, Emissive $\mathrm{ZnO}$-graphene quantum dots for white-light-emitting diodes, Nat. Nanotechnol. 7 (2012) 465-471.

[31] F.L. Kuo, Y. Li, M. Solomon, J. Du, N.D. Shepherd, Workfunction tuning of zinc oxide films by argon sputtering and oxygen plasma: an experimental and computational study, J. Phys. D: Appl. Phys. 45 (2012) 065301-065307.

[32] C. Tao, J. Wang, S. Qin, Y. Lv, Y. Long, H. Zhu, Z. Jiang, Fabrication of pHsensitive graphene oxide-drug supramolecular hydrogels as controlled release systems, J. Mater. Chem. 22 (2012) 24856-24861.

[33] Y.C. Chena, K. Katsumata, Y.H. Chiua, K. Okada, N. Matsushita, YJ. Hsua, ZnOgraphene composites as practical photocatalysts for gaseous acetaldehyde degradation and electrolytic water oxidation, Appl. Catal. A 490 (2015) 1-9.

[34] T. Lv, L. Pan, X. Liu, T. Lu, G. Zhu, Z. Sun, Enhanced photocatalytic degradation of methylene blue by ZnO-reduced graphene oxide composite synthesized via microwave-assisted reaction, J. Alloys Compd. 509 (2011) 10086-10091.

[35] S. Vadivel, D. Maruthamani, B. Paul, S.S. Dhar, H.A. Yangjeh, S. Balachandran, B. Saravanakumar, A. Selvakumar, K. Selvam, Biomolecule-assisted solvothermal synthesis of $\mathrm{Cu}_{2} \mathrm{SnS}_{3}$ flowers/RGO nanocomposites and their visible-lightdriven photocatalytic activities, RSC Adv. 6 (2016) 74177-74185.

[36] X. Zhang, T. Peng, S. Song, Recent advances in dye-sensitized semiconductor systems for photocatalytic hydrogen production, J. Mater. Chem. A 4 (2016) 2365-2402.

[37] G.K. Pradhan, D.K. Padhi, K. Parida, Fabrication of $\alpha-\mathrm{Fe}_{2} \mathrm{O}_{3}$ nanorod/RGO composite: a novel hybrid photocatalyst for phenol degradation, ACS Appl. Mater. Interfaces 5 (2013) 9101-9110.

[38] N. Badr, K.A. Al-Qahtani, Treatment of wastewater containing arsenic using Rhazya stricta as a new adsorbent, Environ. Monit. Assess. 185 (2013) 9669-9681.

[39] K. Vasanth Kumar, Linear and non-linear regression analysis for the sorption kinetics of methylene blue onto activated carbon, J. Hazard. Mater. B 137 (2006) 1538-1544.

[40] A. Afkhami, R. Moosavi, Adsorptive removal of Congo Red, a carcinogenic textile dye, from aqueous solutions by maghemite nanoparticles, J. Hazard. Mater. 174 (2010) 398-403.

[41] W.C. Wanyonyi, J.M. Onyari, P.M. Shiundu, Adsorption of Congo Red Dye from aqueous solutions using roots of Eichhornia crassipes: kinetic and equilibrium studies, Energy Procedia 50 (2014) 862-869.

[42] S. Mahdavi, M. Jalali, A. Afkhami, Removal of heavy metals from aqueous solutions using $\mathrm{Fe}_{3} \mathrm{O}_{4}, \mathrm{ZnO}$, and $\mathrm{CuO}$ nanoparticles, J. Nanopart. Res. 14 (2012) 846.

[43] A.O. Dada, A.P. Olalekan, A.M. Olatunya, O. Dada, Langmuir, Freundlich, Temkin and Dubinin-Radushkevich, isotherms studies of equilibrium sorption of Zn2+ unto phosphoric acid modified rice husk, IOSR J. Appl. Chem. 3 (2012) $38-45$. 
[44] S. Qian, S. Zhang, Z. Huang, M. Xiao, F. Huang, Preconcentration of ultra-trace copper in water samples with nanometer-size $\mathrm{TiO}_{2}$ colloid and determination by GFAAS with slurry sampling, Microchim. Acta 166 (2009) 251-254.

[45] V. Chantawong, N.W. Harvey, V.N. Bashkin, Comparison of heavy metal adsorptions by Thai Kaolin and Ballclay, Water Air Soil Pollut. 148 (2003) 111 125.

[46] O. Yavuz, Y. Altunkaynak, F. Güzel, Removal of copper, nickel, cobalt and manganese from aqueous solution by kaolinite, Water Res. 37 (2003) 948-952.

[47] J. Wang, X. Ma, G. Fang, M. Pan, X. Ye, S. Wang, Preparation of iminodiacetic acid functionalized multi-walled carbon nanotubes and its application as sorbent for separation and preconcentration of heavy metal ions, J. Harzard. Mater. 186 (2011) 1985-1992.
48] S.H. Huang, D.H. Chen, Rapid removal of heavy metal cations and anions from aqueous solutions by an amino-functionalized magnetic nano-adsorbent, J. Harzard. Mater. 163 (2009) 174-179.

[49] B. Yu, J. Xu, J.H. Liu, S.T. Yang, J. Luo, Q. Zhou, J.W.R. Liao, H. Wang, Y. Liu, Adsorption behavior of copper ions on graphene oxide-chitosan aerogel, J. Environ. Chem. Eng. 1 (2013) 1044-1050.

[50] F. Fang, L. Kong, J. Huang, S. Wu, K. Zhang, X. Wang, B. Sun, Z. Jin, J. Wang, X. Huang, J. Liu, Removal of cobalt ions from aqueous solution by an amination graphene oxide nanocomposite, J. Hazard. Mater. 270 (2014) 1-10.

[51] A. Tayyebi, M. Outokesh, Supercritical synthesis of a magnetite-reduced graphene oxide hybrid with enhanced adsorption properties toward cobalt \& strontium ions, RSC Adv. 6 (2016) 13898-13913. 